This item was submitted to Loughborough's Research Repository by the author.

Items in Figshare are protected by copyright, with all rights reserved, unless otherwise indicated.

\title{
Usability problem reports for comparative studies: consistency and inspectability
}

\section{PLEASE CITE THE PUBLISHED VERSION}

PUBLISHER

(c) Taylor \& Francis

\section{VERSION}

AM (Accepted Manuscript)

\section{LICENCE}

CC BY-NC-ND 4.0

\section{REPOSITORY RECORD}

Vermeeren, Arnold P.O.S., Jelle Attema, Evren Akar, Huib de Ridder, Andrea J. von Doorn, Cigdem Erbug, Ali E. Berkman, and Martin Maguire. 2019. "Usability Problem Reports for Comparative Studies: Consistency and Inspectability”. figshare. https://hdl.handle.net/2134/4252. 
This item was submitted to Loughborough's Institutional Repository (https://dspace.lboro.ac.uk/) by the author and is made available under the following Creative Commons Licence conditions.

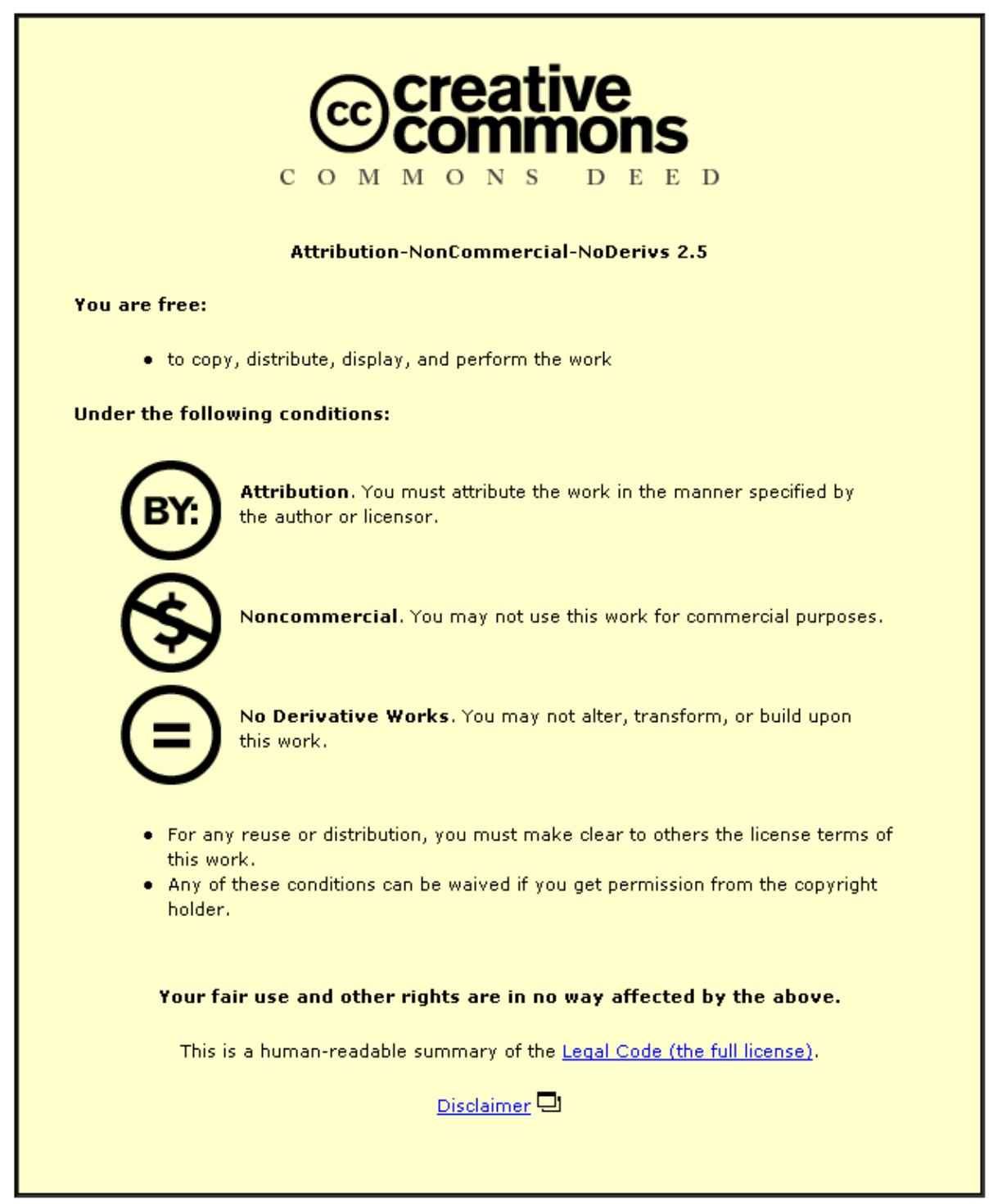

For the full text of this licence, please go to: http://creativecommons.org/licenses/by-nc-nd/2.5/ 


\title{
Usability problem reports for comparative studies: consistency and inspectability
}

\author{
Arnold P.O.S. Vermeeren, Jelle Attema, Evren Akar, \\ Huib de Ridder and Andrea J. van Doorn \\ Delft University of Technology, The Netherlands
}

Çiğdem Erbuğ and Ali E. Berkman

Middle East Technical University, Turkey

Martin C. Maguire

Loughborough University, United Kingdom

\begin{abstract}
The present study explores issues of consistency and inspectability in usability test data analysis processes and reports. Problem reports resulting from usability tests performed by three professional usability labs in three different countries are compared. Each of the labs conducted a usability test on the same product, applying an agreed test protocol that was collaboratively developed by the labs. Each lab first analyzed their own findings as they always do in their regular professional practice. A few weeks later, they again analyzed their findings but then everyone applied the same method (SlimDEVAN: a simplified version of DEVAN, a method developed for facilitating comparison of findings from usability tests in an academic setting). It was found that levels of agreement between labs did not improve when they all used SlimDEVAN there was inherent subjectivity in their analyses. It was found that consistency of single analysts in analyzing their data can be improved by using a method like SlimDEVAN. Such methods can also help in making the analysis process and findings more inspectable. Inspectability is helpful in comparative studies based on identified usability problems because it allows for the traceability of findings to original observations, as well as for laying bare the subjective parts of the data analysis.
\end{abstract}




\section{INTRODUCTION}

1.1 Consistency of individual analysts (reliability)

1.2 Consistency of findings across multiple analysts (subjectivity)

1.3 Aim of the study

\section{CASE STUDY}

2.1 Usability tests on an advanced oven interface

The laboratories

The product

Sessions and task scenarios

Participants

2.2 The labs' data analysis procedures

Lab A
Lab B
Lab C

3. THE REFERENCE ANALYSES

3.1 Description of SlimDEVAN

3.2 How the labs learned to use SlimDEVAN

3.3 The labs' Slim DEVAN analyses
Lab A
Lab B
Lab C

\section{COMPARING THE REPORTS}

4.1 Making the reported findings comparable

Procedure and resulting material

Findings on consistency and inspectability

4.2 The comparisons

Comparing problems reported in a lab's subsequent

analyses

Comparing the reported problems across the three labs

\section{DISCUSSION AND CONCLUSION}

5.1 Inspectability

5.2 Consistency

Across-analyst consistency (subjectivity)

Within-analyst consistency

5.3 Follow-up study on causes of (in)consistency

Conclusions

Implications 
Arnold Vermeeren is an industrial design engineer with an interest in usability, user experience and interaction design; he is an Assistant Professor at the faculty of Industrial Design Engineering of Delft University of Technology. Huib de Ridder is sdsd s asfsfasf asfsf asfasf asfasfs asfas asfasfa ags adgag xcvx xcvxcve xcve xcvxc xcvxcev xcvxce xcv xvxcv fsdf sfsdfsd ssd sgsdgsdg sdsdgsd sgsgsd sdgsdsdg sdgsdg sgd sdgsdg sdgsdsdgsd sgsdgsdgsdgsdg dsgsdgsdg. Andrea van Doorn is sdsd s asfsfasf asfsf asfasf asfasfs asfas asfasfa ags adgag xcvx xcvxcve xcve xcvxc xcvxccv xcvxcc xcv xvxcv fsdf sfsdfsd ssd sgsdgsdg sdsdgsd sgsgsd sdgsdsdg sdgsdg sgd sdgsdg sdgsdsdgsd sgsdgsdgsdgsdg dsgsdgsdg. Jelle Attema is sdsd s asfsfasf asfsf asfasf asfasfs asfas asfasfa ags adgag xcvx xcvxcve xcvc xcvxc xcvxccv xcvxcc xcv xvxcv fsdf sfsdfsd ssd sgsdgsdg sdsdgsd sgsgsd sdgsdsdg sdgsdg sgd sdgsdg sdgsdsdgsd sgsdgsdgsdgsdg dsgsdgsdg. Evren Akar is sdsd s asfsfasf asfsf asfasf asfasfs asfas asfasfa ags adgag xcvx xcvxcvc xcve xcvxc xcvxccv Xcvxcc Xcv XvXcv fsdf sfsdfsd ssd sgsdgsdg sdsdgsd sgsgsd sdgsdsdg sdgsdg sgd sdgsdg sdgsdsdgsd sgsdgsdgsdgsdg dsgsdgsdg. Cigdem Erbug is sdsd $\mathrm{s}$ asfsfasf asfsf asfasf asfasfs asfas asfasfa ags adgag xcvx xcvxcve xcve xcvxc xevxcev xcvxcc xev xvxcv fsdf sfsdfsd ssd sgsdgsdg sdsdgsd sgsgsd sdgsdsdg sdgsdg sgd sdgsdg sdgsdsdgsd sgsdgsdgsdgsdg dsgsdgsdg. Ali Berkman is sdsd $\mathrm{s}$ asfsfasf asfsf asfasf asfasfs asfas asfasfa ags adgag xcvx xevxcve xeve xevxc xevxecv xevxce xcv xvxcv fsdf sfsdfsd ssd sgsdgsdg sdsdgsd sgsgsd sdgsdsdg sdgsdg sgd sdgsdg sdgsdsdgsd sgsdgsdgsdgsdg dsgsdgsdg. Martin Maguire is sdsd s asfsfasf asfsf asfasf asfasfs asfas asfasfa ags adgag xcvx xcvxcve xcvc xcvxc xcvxcev xcvxce xcv xvxcv fsdf sfsdfsd ssd sgsdgsdg sdsdgsd sgsgsd sdgsdsdg sdgsdg sgd sdgsdg sdgsdsdgsd sgsdgsdgsdgsdg dsgsdgsdg.

\section{INTRODUCTION}

Usability testing often takes place in the context of product development processes (for software, websites, electronic products, etc.) as a way of getting feedback on product usability. In some specific cases, findings from multiple usability tests need to be systematically compared. For example, in academic settings comparative studies may be conducted to study methodological issues of user evaluations (e.g., Jacobsen, Hertzum \& John, 1998; Vermeeren, 1999; Cockton, Lavery \& Woolrych, 2002; Molich, Ede, Kaasgaard \& Karyukin, 2004; Vermeeren, Bekker, van Kesteren \& de Ridder, 2007); in other settings they may be conducted to compare usability of competing designs or design proposals (e.g., Park and Lim, 1999; Hoenderdos, Vermeeren, Bekker, \& Pierik, 2002) and in international product development settings they may be 
conducted to determine cultural differences in product use or usability (e.g., Daams \& Hariandja, 2006, Noiwana \& Norcio, 2006).

For being able to properly draw conclusions based on comparisons of findings from different test conditions, one needs to be reasonably sure that identified differences in findings can be attributed to differences in conditions, rather than to for example inconsistencies in data analysis or idiosyncratic focus or interpretations of an evaluator. Issues like these are often referred to as issues of reliability and subjectivity (or objectivity) respectively. In the present study, three labs in three different countries conducted usability tests on the same product, applying an agreed test protocol and then (independent from each other) analyzed their data and reported their findings. Based on the labs' problem reports, issues of reliability and subjectivity are studied with a focus on the usability labs' data analyses and reporting of results. Before proceeding to the introduction of the case study, the following section will discuss issues of reliability and subjectivity in more depth.

\subsection{Consistency of individual analysts (reliability)}

Hartson, Andre \& Williges (2001) discuss a number of quality criteria for Usability Evalution Methods (UEMs) including: thoroughness, validity, effectiveness, and reliability. They define how to measure thoroughness, validity and effectiveness based on counting usability problems. However, they do not precisely define the concept of reliability in terms of how to calculate it; Hartson, Andre \& Williges (2001) define reliability primarily in terms of evaluator agreement as "... a measure of the consistency of usability testing results across different users of the UEMs (evaluators)." However, they also speak about individual reliability, stating that "... In most UEMs, low individual reliability means high variability among evaluators, which means that merging results over a group of evaluators will give higher overall thoroughness." Thus individual reliability seems to refer to evaluations conducted by one single evaluator; presumably they refer to consistency of UEM results in cases where an evaluator applies a UEM multiple times on the same material (i.e., within-evaluator consistency).

In line with Hartson, Andre \& Williges' definition, Guba and Lincoln (1989) state that (in research starting from a positivistic paradigm) the establishment of reliability "... typically rests on replication, assuming that every repetition of the same or equivalent instruments to the same phenomena will yield similar measurements." However, they also state that in research based on a naturalistic research paradigm, where by definition measurements cannot be exactly repeated, the issue of reliability (or dependability, as it is often referred to in that context) is dealt with mainly by making sure that the used process is "... an established, trackable, and documentable process," so 
that outside reviewers "... can explore the process, judge the decisions that were made, and understand what salient factors in the context led the evaluator to the decisions and interpretations made (Guba and Lincoln, 1989, p. 242)."

Kanis (1993) performed an extensive literature study to determine how the term reliability is used in two important constituent disciplines of human factors and ergonomics, namely technical sciences and social sciences. As a result of his inquiry he proposed "... to maintain a clear-cut distinction between random variation and systematic deviance in outcomes of measurements" and to use the term reproducibility rather than reliability. According to Kanis (1993), reliability (or reproducibility) should deal with random variation, rather than with systematic deviance.

In case of usability test data analyses based on extracting usability problems, the analyst forms part of the instrument for identifying problems; after all, ultimately, it is the analyst who judges whether an event is considered problematic or not. Presumably, analyst judgments are largely based on individual expertise, experiences, and ability to empathize with users. In such a context reliability concepts based on 'repeated measurements' are problematic as they assume that in the repeated analysis, the analyst has forgotten everything about the previous analysis, and has not gained any relevant, additional knowledge or experience affecting his/her perception of interactions (which will probably never be completely true). For the same reason it is also questionable to what extent differences in findings from multiple analyses of a single analyst can be regarded as 'random variation' (cf. Kanis, 1993). Therefore, in the remainder of this article the term 'reliability' will be avoided and the issue will be dealt with mainly in terms of 'consistency of individual analysts'.

In this article, consistency of individual analysts will be dealt with by using the repeated measures approach (within-analyst consistency), but taking into account Guba and Lincoln's (1989) advice to use established, documentable and traceable processes.

\subsection{Consistency of findings across multiple analysts (subjectivity)}

According to Guba and Lincoln (1989) objectivity is "...concerned with assuring that data interpretations and outcomes of inquiries are rooted in contexts and persons apart from the evaluator and are not simply figments of the evaluator's imagination." In addition, they state that in a naturalistic research paradigm one may not assume that methods can prevent the inquirer 
to (even inadvertently) introduce subjectivity in findings. Instead, assurances of integrity of findings are rooted in the data themselves. In other words, the starting point is that (at least some degree of) subjectivity is acknowledged in data analysis and should be dealt with properly. In Guba and Lincoln's view this means that both the original data and the processes used to compress these data should be available to be inspected and confirmed by outside reviewers of the study. For this they adopt a criterion of confirmability rather than objectivity. Probably, the term inspectability would be more appropriate, as the products and processes should be available for inspection (not only for confirmation, but also for falsification).

Because in usability test data analyses based on extracting usability problems the analyst forms part of the measurement instrument, Hartson, Andre \& Williges' (2001) definition of reliability as "... a measure of the consistency of usability testing results across different users of the UEMs (evaluators)" (and analyst agreement in general) should be seen as primarily dealing with issues of subjectivity/objectivity, rather than with reliability purely. Findings from a number of studies investigating the so-called evaluator effect seem to confirm this notion. The evaluator effect (e.g., Hertzum \& Jacobsen, 2001; Vermeeren, van Kesteren \& Bekker, 2003; Law \& Hvannberg, 2004) is the effect that if several evaluators analyze the same video recorded user test sessions there is a considerable lack of overlap in their findings. The findings from the studies suggest that no matter how careful, structured and detailed the analysis is, if it involves qualitative analyses there is a considerable subjective component in it (e.g., see Vermeeren, van Kesteren \& Bekker, 2003). This suggests that, also in case of user test data analyses, assurances for objectivity cannot be rooted entirely in the method used and its subjectivity will have to be dealt with somehow.

In the remainder of this article, issues of subjectivity and objectivity will be dealt with by using measures of analyst agreement as well as by keeping in mind Guba \& Lincoln's (1989) advice that it should always be possible to trace back on what data the findings are based and how the data transformed from primary observations into findings (i.e. findings should be inspectable).

\subsection{Aim of the study}

Aim of the study presented in this article is to investigate issues of consistency and inspectability of data analyses and reports from usability tests based on extracting usability problems. For that purpose usability tests were conducted by labs in three different countries. They all tested the same product, applying the same test protocol. Subsequently, each individual lab analyzed their data and reported about it. After the labs had finished writing their reports, they were asked to re-analyze their data a few weeks later, now 
applying (a simplified version of) the DEVAN tool (Vermeeren, den Bouwmeester, Aasman \& de Ridder, 2002); the DEVAN tool was originally developed for improving an analyst's consistency in data analysis, for documenting the analysis procedures and for making findings inspectable (in order to facilitate recognition of subjectivity in each of the data analysis stages).

The labs' reports formed the basis for making various comparisons. The first step to making comparisons was to compile a 'complete' master list of usability problems from the labs' reports. As problem formulations in lab reports were sometimes too ambiguous or incomplete to understand problems in enough detail for direct comparison, there proved to be a need to track back reported problems to their original sources. Experiences in trying to do so, have provided insight into issues of inspectability of the labs' reported findings. Based on the master problem list, measures of consistency (withinanalyst consistency, as well as analyst agreement) were calculated. Inconsistencies in findings were analyzed in more depth by trying to trace back findings to the original data (inspectability) and identifying possible causes of differences. Identified causes of differences indicate whether these are due to issues of inconsistency of individual analysts or of inconsistency between multiple analysts. Also, those procedures that had been described in enough detail and those findings that were inspectable enough, indicated at what stage in the data analysis process inconsistencies (either within individual analysts or between analysts) occurred.

In the next section, the usability tests conducted by the three labs are described, along with the analyses they performed and the way they reported their findings. Subsequently, in section 3, the reference analysis procedure (the simplified version of the DEVAN procedure) is explained, along with the labs' report formats that resulted from applying it. Section 4 discusses the procedure, experiences and results of comparing the reported findings and reports the identification of relevant analysis and report characteristics. In section 5 the results are discussed and implications for data analyses and usability problem reports in practice are drawn.

\section{CASE STUDY}

This section introduces the usability tests that the labs conducted. It then discusses the ways in which the labs analyzed the user test data according to their usual professional practices and shows examples of the used report formats. 


\subsection{Usability tests on an advanced oven interface}

\section{The laboratories}

The laboratories that conducted the user tests were UTEST at the Middle East Technical University in Ankara (Turkey), the WIT-lab at Delft University of Technology in the Netherlands, and ESRI at Loughborough University in the United Kingdom.

At the time of the test, UTEST was a relatively new usability laboratory within Middle East Technical University. UTEST provides services to industry and promotes academic studies. The collaboration with industry consists of both research and consultancy activities embracing military and consumer products as well as software and electronic appliances. Evaluators sitting in the control room of the lab can observe users in the test room through monitors and a one-way mirror. Remote-controlled and portable digital cameras are used to record user performance and observational software is used for data analysis. The research team consists of experts having diverse academic backgrounds.

The WIT-lab (Laboratory for Work and Interaction Technology) is a laboratory at Delft University of Technology, for both commercial usability services and for the support of research and educational projects. At the time of the study it had more than ten years of experience in commercial usability services. The lab has two test rooms, an evaluation room and a control room. Staff members have a background in organizational psychology, experimental psychology and systems engineering.

ESRI (The Ergonomics and Safety Research Institute) is located within Loughborough University and was formed from two institutes on ergonomics and human factors (HUSAT and ICE) dating back to the early 1970s. ESRI provides research, consultancy and teaching in the area of human interaction with computer systems, products and advanced technology. The ESRI usability laboratory is fitted with audio-visual equipment for testing product usability and is split into two areas: a user-work area and an evaluator's control room from which users can be monitored directly through a one-way mirror. Remote-controlled and portable video cameras are used to capture the users' interactions and performance with the product. The camera images are mixed and stored on tape for analysis. ESRI staff has a background in ergonomics, computer science and psychology and has more than ten years of experience in offering usability services in a commercial context.

\section{The product}

The product that was used as the object of evaluation was a prototype of an advanced oven interface panel. The interface consisted of a combination of an 
LCD display with two push buttons and a rotary knob; at all times during product use, the function of each button is shown on the display. In the test room a prototype of the complete oven casing was shown, with a non functioning interface on it. Next to it was a cardboard mockup with a functioning interface on it (see figure 1).

Figure 1. A participant interacting with a mockup of the oven's interface panel.

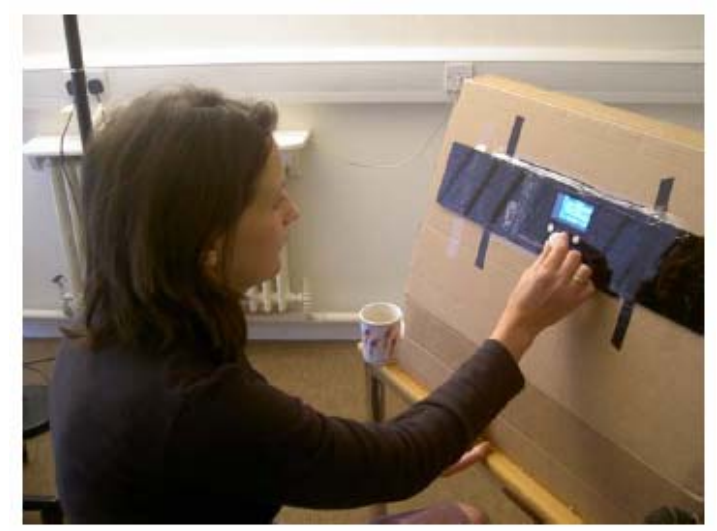

\section{Sessions and task scenarios}

Test protocols were collaboratively developed by the three labs. Scenarios were defined prescribing what tasks each participant had to perform with the control panel; these scenarios covered most of the functionality of the oven and were phrased as real-life contextual goals to be reached by participants; for example: "Suppose the test room is the kitchen of a friend of yours. You enter the kitchen and you see that it is filled with smoke and that the smoke is coming from the oven. You see that the oven is working...something is cooking inside. Please go in and try to stop the cooking." and "For some special dishes the oven knows how it has to be set. Now suppose that you want to grill a large sized fish. See if the oven knows this recipe and if it does then start grilling the fish."

After each task scenario, follow-up questions were asked for clarification of actions with unclear intentions (e.g., "what did you expect would happen when you pressed that button?" or "what did you think had happened when you saw that screen appear?"). On average, sessions lasted about one hour. 


\section{Participants}

Sampling of participants was done according to the manufacturer's market profile. In each of the three countries eight participants took part in the trials ( 6 female and 2 male, age varying between 20 and 55 years). All participants were regular cooks and part of a family of 3 to 5 members. They all had either recently purchased an oven or had recently considered the possibility of purchasing one. Other characteristics included: they are full-time or part-time employees; they are indigenous individuals, they cook for themselves at least 4 times a week; they not always cook ready-made meals; they live in a city or town environment.

\subsection{The labs' data analysis procedures}

For each of the labs, the lab's data analysis procedure is described below.

\section{Lab A}

In lab $\mathrm{A}$ two researchers participated in the test. Researcher 1 acted as facilitator, conducting the test sessions (providing participants with tasks, helping them if necessary and asking questions). During task performance, researcher 2 (acting as observer) watched participants performing tasks, took some notes and sometimes discussed with two student observers what exactly was happening. After the sessions, researcher 1 (the facilitator) watched the video recorded task performance sessions and wrote notes about the sessions in a format as shown in appendix 1. Researcher 2 (the observer) and the two student observers did not take part in the analysis of the video recorded sessions.

\section{Lab B}

In lab $\mathrm{B}$, two researchers were involved in the test. Researcher 1 (the observer) manually logged sessions in real time during task performance and interviews, using dedicated database software. Logs were automatically timestamped and linked to the digital video recordings. Appendix 2 shows an example $\log$ (NB. The logs were not part of the reports that were handed in and thus could not be used in the comparisons). Researcher 2 acted as facilitator during the test, but did not take part in the data analysis.

After the sessions, researcher 1 went through the event logs (during the analysis video recordings were automatically kept synchronized with the log files) and assigned so called findings to logged events. Findings were then categorized according to tasks. Subsequently, for each finding a paragraph discussing the weight of the findings was written, as well as a paragraph 
providing suggestions for solutions. Weight and suggestions for solutions were inspired by going through the additional non-finding events in the log files. Finally, for each finding a number of example events was selected for inclusion in the report. This resulted in a report format as shown in appendix 3.

Lab C

In lab $\mathrm{C}$, two researchers participated in the test: researcher 1 acted as facilitator, researcher 2 as observer. During task performance, researcher 2 (with no strong understanding of the product interface) took notes on a printed version of the task protocol, focusing mainly on timing of tasks, as well as on key comments and actions of participants. Researcher 1 took notes on a printed version of the task protocol, using their own defined abbreviations. The notes from both researchers were discussed amongst them and were then combined and typed up as a single record of each session.

The data analysis was jointly done by both researchers. Subsequently, researcher 1 wrote a report based on the combined notes. Video tapes were now and then used as a reference during the process. Appendix 4 shows examples of lab C's report.

\section{THE REFERENCE ANALYSES}

One to two months after the labs had reported their findings, the videotaped sessions were analyzed again. This time a prescribed, detailed analysis was performed, using SlimDEVAN (a simplified version of DEVAN; Vermeeren, den Bouwmeester, Aasman \& de Ridder (2002) as a reference to compare the initial analysis to. Below, SlimDEVAN will first be explained, followed by a brief description of how the labs got acquainted with it. Then the labs' SlimDEVAN analyses will be presented together with the report formats they resulted in.

\subsection{Description of SlimDEVAN}

SlimDEVAN is a checklist-based approach to user test data analysis. It is a simplified version of the DEVAN technique for video data analysis (Vermeeren, den Bouwmeester, Aasman \& de Ridder, 2002). Main differences between DEVAN and SlimDEVAN lie in the way in which overviews of interactions are made. In case of DEVAN, the procedure for arriving at the overviews as well as the format for the overviews are prescribed in much detail; in case of SlimDEVAN decisions on these issues are largely left to the individual analyst, but advice and constraints are given (see below). Both DEVAN and SlimDEVAN make use of a checklist. The 
checklist (see figure 2) aids in detecting events that signal the existence of interaction difficulties by defining such events. In this context, the term difficulty does not necessarily refer to a complete halt in task performance. For example, hesitations before (or frustration after) successful task performance are also regarded as difficulties, as are erroneous actions that are corrected instantaneously. The use of the checklist stimulates that evaluators use the same definition of what constitutes an interaction difficulty. Moreover, it makes the analysis process more explicit. The DEVAN checklist is based on Lavery, Cockton and Atkinson's (1997) definition of usability problems which describes, in general terms, the behavioral and outcome consequences of usability problems. The SlimDEVAN checklist is basically the same as the DEVAN checklist (Vermeeren, den Bouwmeester, Aasman \& de Ridder, 2002) but was slightly adapted based on experiences in other projects (e.g., Barendregt and Bekker, 2005; Vermeeren, Bekker, van Kesteren and de Ridder, 2007).

Basically, two types of observations are distinguished within the checklist. These are:

- physical actions performed on the product (i.e. actions performed on the products' control elements);

- expressions: (verbal) utterances from users, as well as body language (i.e., facial expressions, gestures, etc.).

The checklist assumes that both types of difficulty signals can be found at several stages of performing an action: (a) prior to physically performing the action (e.g., user hesitates before acting), (b) during the physical performance of an action (e.g., mis-pressing a button), (c) directly following an action (e.g., exclamation of surprise after seeing the system's reaction to an action) or (d) later in a session (e.g., when after continuing with other actions the user suddenly notices a preceding erroneous action and corrects it).

The SlimDEVAN approach works best if the analysis starts from a session $\log$ that specifies time-stamped actions on the product, as well as (verbal, gestural, facial) user expressions. The procedure is to go through a log file (or if desired also review parts of video taped sessions) and search for the types of events as defined in the checklist (the so-called difficulty signals). Codes for detected difficulty signal events are added to the session logs (thereby making the events time-stamped). Subsequently, for each participant, a list of difficult moments is created, preferably with time-stamps added. A single moment of difficulty can be signaled by multiple event types at a time (i.e., a single usability problem can be identified based on multiple signaling events). Figure 3 shows examples of how moments of difficulties can be specified (these examples are taken from the SlimDEVAN user manual (Vermeeren, 2003)). 


\subsection{How the labs learned to use SlimDEVAN}

The first time the labs heard about DEVAN was during the first project meeting with all labs. In that meeting they were told about the existence of DEVAN and were provided with copies of the article in which the tool was first introduced (Vermeeren, den Bouwmeester, Aasman \& de Ridder, 2002). At that time, however, DEVAN was not considered feasible for use in the project, due to its time-consuming nature. At a later stage, after the second project meeting, the idea of using SlimDEVAN arose.

A brief description of how SlimDEVAN differed from DEVAN was sent to all partners by email, along with a few paragraphs of information about what actions would be required from them if they would be willing to use SlimDEVAN. After the three labs agreed on using SlimDEVAN, a user manual (Vermeeren, 2003) was written. The user manual included a separate checklist reference card specifying the SlimDEVAN codes for difficulty signals. In a third meeting the labs were provided with a copy of the user manual. In addition, the procedures for using it were orally explained and questions were answered in order to clarify what the labs could expect while using it. It was suggested to the labs that they could send part of the results of the analysis of one participant's task performance to the first author of the present article (who had not been involved in conducting the tests). In this way, they would have the opportunity to have their analyses checked for misunderstanding SlimDEVAN. Lab C made use of this possibility. 


\title{
Figure 2. The SlimDEVAN checklist (at the time of the test the word breakdown was used in the checklist, as a synonym for the word difficulty in the present article).
}

\author{
Breakdown signals in the form of physical actions performed on the product \\ (code, short description: definition)
}

ACT, wrong action: An action does not belong in the correct sequence of actions, an action is omitted from the sequence, an action within the sequence is replaced by another action, or actions within the sequence are performed in reversed order DISC, discontinued action: User points at function as if to start executing it, but then does not, or user stops executing action, before it is finished.

EXE, execution problem: Execution of action not done correctly or optimally.

REP, repeated action: An action is repeated with exactly the same effect.

CORR, corrective action: An action is corrected with a subsequent action (or sequence of actions), or an action is undone.

STOP, task stopped: User starts new task, before having successfully finished the current task.

Breakdown signals in the form of utterances (verbal, sound) or body language (facial expressions, gestures)

(code, short description: definition):

PER, perception problem: User indicates (in words or behavior) not to be able to hear or see something clearly.

INTN, wrong goal or intention: User formulates a goal that cannot be achieved with the product or that does not contribute to achieving the task goal; or user (verbally) specifies an action that $\mathrm{s}$ /he think is needed in order to progress towards the goal, but the specified action is not correct (indicating wrong user intention).

PUZZ, puzzled (before an action): User indicates (in words or behavior) not to know how to perform the task or what action is needed for it, or not to be sure whether a specific action is needed or not.

RAND, random actions: User indicates (in words or behavior): that the current action(s) are chosen randomly

SEARCH, searching for a function (but not finding it): User indicates (in words or behavior): not being able to locate a specific function

DIFF, execution difficulty: User indicates (in words or behavior) having physical problems in executing an action, or that executing the action is difficult or uncomfortable

DSF, doubt, surprise, frustration (after an action): User indicates (in words or behavior) not to be sure whether an action was executed properly, not to understand an action's effect, to be surprised by an action's effect or that the effect of an action was unsatisfactory or frustrated the user.

WEX, wrong explanation (after an action): User formulates an explanation for something that happens, but this explanation is not correct; or user formulates an interpretation for displayed feedback, but this interpretation is not correct.

REC, recognition of error or of misunderstanding: User indicates (in words or behavior) to recognize a preceding error, or to understand something previously not understood QUIT, quits task: User indicates (in words or behavior) to recognize that the current task was not finished successfully, but continues with a subsequent task; or user indicates (in words or behavior) that $\mathrm{s} /$ he thinks a task was successfully finished and continues with a subsequent task, (whereas in fact the task was not finished successfully). 
Figure 3. Part of a usability list, as an example format for the evaluators (at the time of the test the word breakdown was used as a synonym for the word difficulty).

\begin{tabular}{|c|c|c|}
\hline $\begin{array}{l}\text { Time stamp and } \\
\text { signal codes }\end{array}$ & $\begin{array}{l}\text { Free-form breakdown } \\
\text { description }\end{array}$ & $\begin{array}{l}\text { Inferences about what } \\
\text { design elements may } \\
\text { have caused the } \\
\text { breakdown to occur. }\end{array}$ \\
\hline $\begin{array}{l}0: 02: 40 \\
\text { ACT } \\
\text { DSF } \\
0: 04: 20 \\
\text { CORR }\end{array}$ & $\begin{array}{l}\text { User wants to change Time } \\
\text { style, and clicks at the time } \\
\text { in the bottom right corner. } \\
\text { Apparently, he expects that } \\
\text { time style settings can be } \\
\text { found there. He should have } \\
\text { gone to regional settings in } \\
\text { the control panel. }\end{array}$ & $\begin{array}{l}\text { At two places there } \\
\text { are settings related to } \\
\text { time. At only one of } \\
\text { those places it is } \\
\text { possible to change } \\
\text { Time Style. User } \\
\text { expects it to be where } \\
\text { the clock is, but it isn't. }\end{array}$ \\
\hline $\begin{array}{l}0: 03: 10 \\
\text { INTN } \\
\text { ACT }\end{array}$ & $\begin{array}{l}\text { User expects to find Time } \\
\text { style settings in the Time } \\
\text { Zone tab of the Date/Time } \\
\text { Properties, instead of in the } \\
\text { Regional Settings in the } \\
\text { Control Panel. }\end{array}$ & $\begin{array}{l}\text { At two places there } \\
\text { are settings related to } \\
\text { time. At only one of } \\
\text { those places it is } \\
\text { possible to change } \\
\text { Time Style. User } \\
\text { expects it to be where } \\
\text { the clock is, but it isn't. }\end{array}$ \\
\hline
\end{tabular}

\subsection{The labs' SlimDEVAN analyses}

Below, each lab's implementation of the SlimDEVAN reference analysis is described.

\section{Lab A}

In case of lab A, two researchers participated in the SlimDEVAN analysis. These were the same researchers as in the lab specific approach. First, the researchers together analyzed part of one participant's session that seemed difficult to analyze and discussed their implementation of SlimDEVAN. In this way they developed a common understanding about the use of it. Then, each researcher watched the videos of four participants and took notes using identical table formats (see appendix 5 for an example). The checklist card was used as a reference during the analysis. In addition, researcher 2 (the observer) sometimes compared fragments from different sessions to ensure better consistency. Also, researcher 2 analyzed one (difficult to understand) session twice to feel more confident about the findings. Subsequently, researcher 2 went through all typed up tables of both researchers to check for 
consistency in assigning codes. This led to only a few changes in the tables of researcher 2. Finally, for each participant a usability problem list was created. This was done by researcher 1. Appendix 6 shows an example of the format used for reporting the problems.

\section{Lab B}

In case of lab B, researcher 1 (the observer) did the analysis. The SlimDEVAN analysis started from the log files made in the lab's initial analysis. Appendix 7 shows an example of part of a session log made by lab B. Appendix 8 shows an example report format ${ }^{1}$.

The researcher went through all log files on the computer and while going through them, the video recordings were automatically kept synchronized with the $\log$ files. The dedicated software for logging was modified to allow for entering SlimDEVAN codes as markers into the log files. An additional marker called Comment was added for difficulty signals found in the interviews and for comments from the researcher (e.g., ideas for solutions to problems). As the video recordings ran along with the log files, it was possible for the researcher to especially focus on those parts of the video for which no loggings were made, as well as on parts of the log files for which it was not clear what exactly had been observed. At several points the original log files (from the lab specific analysis) proved to be incomplete.

Subsequently, the log files (including the SlimDEVAN codes) were filtered such that a list of (SlimDEVAN) coded events was created. Findings were then defined based on the coded events and multiple events could be linked to a single finding. In the next step of the analysis, the findings were grouped into categories that emerged during the process itself (e.g., consistency, changing parameters during cooking, etc.). Categories in turn were grouped into chapters. Summaries of the findings reported in a chapter were made and frequencies of findings were related to variables like participant, participant characteristics or tasks, to get more insight into those situations in which problems occurred (in the report this was referred to as validity). Descriptions of loosely judged problem severity were written as well as suggestions for solutions. In writing about validity, severity and suggestions, the researcher especially searched for inspiration by going through those events that had not resulted in findings.

\footnotetext{
${ }^{1}$ Although $\log$ files with SlimDEVAN codes were made for all eight participants, lab B accidentally reported problems for only six participants.
} 
Lab C

In case of lab $\mathrm{C}$, both researchers together watched the tape of one of the participants and discussed it in relation to the SlimDEVAN checklist. Researcher 2 then watched the video of that participant again and wrote down actions and times of actions. Subsequently, researcher 2 watched the video again to add participant expressions to the action log, as well as to assign SlimDEVAN codes to events. Researcher 1 followed the same procedure for the other seven participants. Notes were typed up by each of the researchers in a format as shown in appendix 9. Finally, researcher 1 used the format as shown in appendix 10 to create a list of usability problems and indicate which participants experienced each problem.

\section{COMPARING THE REPORTS}

In this section, the protocols for making comparisons are presented along with the results of the comparisons. First, in section 4.1, it will be discussed how the findings in the labs' reports were re-formatted into a form that allowed for making comparisons: across labs, as well as across each lab's subsequent analyses. Issues of inspectability of reports as experienced in this process are discussed. Then, in section 4.2 , it is discussed what exact measures are used for making comparisons.

\subsection{Making the reported findings comparable}

\section{Procedure and resulting material}

Figure 4 illustrates the process of making the reported findings comparable. Starting points were the reports with problem descriptions as they were handed in by the labs (figure 4, blocks at the top). The reports were used and compared without any further clarification and explanations by the labs. The comparer (the first author of the present article, who had not been involved in conducting or analyzing any of the test sessions) read through all reports and (as much as possible) annotated each moment of difficulty reported in a problem description with a unique identification number (id). However, this proved not always to be possible. In some cases, problem descriptions referred to $n$ unspecified users having experienced the problem, or contained no reference to any specified user or specified number of users at all. Figure 5 specifies the applied decision procedure for assigning ids to moments of difficulty. 
Figure 4. Problem descriptions in lab reports (blocks at the top) were reformatted into difficulty records (blocks in the middle). Then similar moments of difficulty were grouped in order to arrive at a master list of usability problems (block at the bottom).

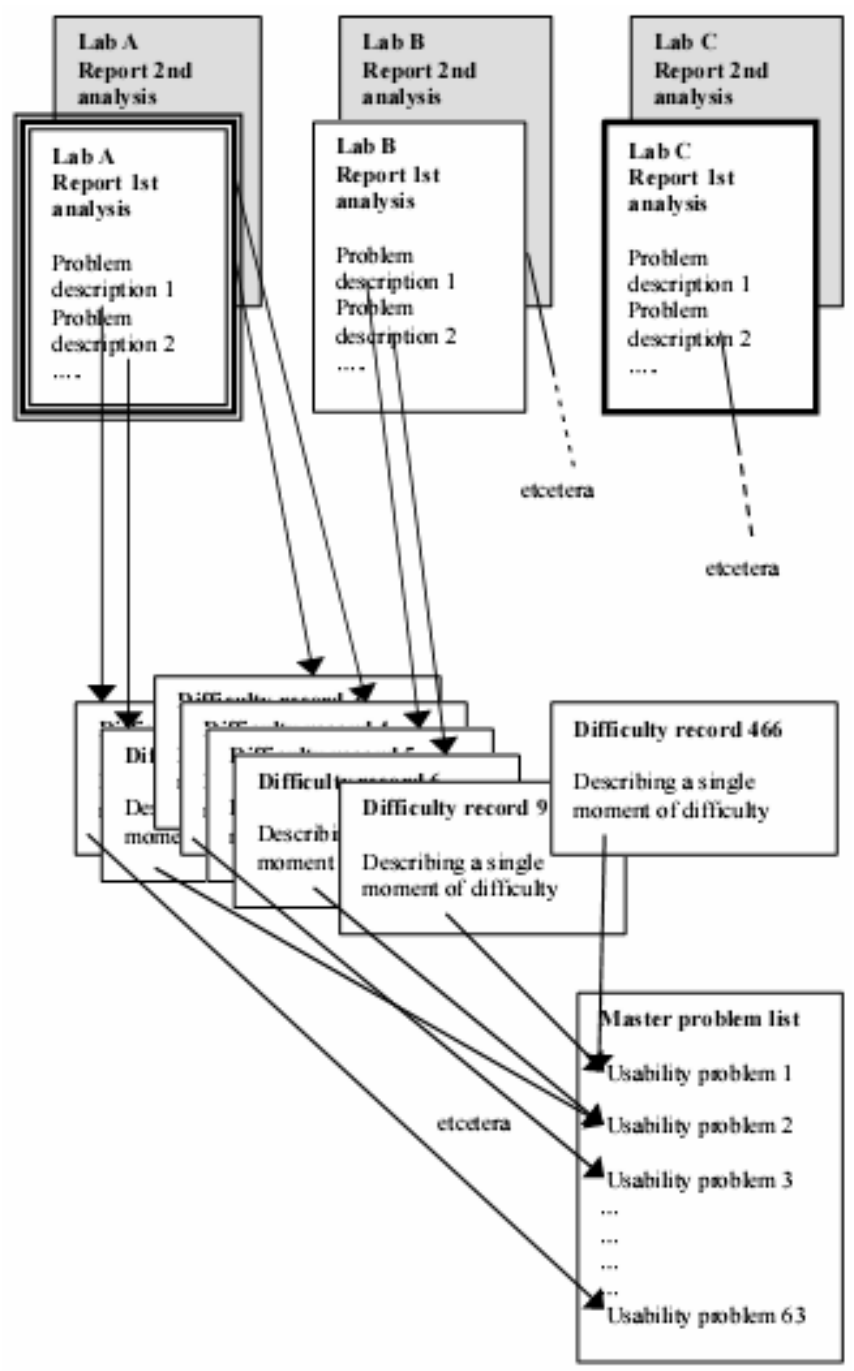


Figure 5. Decision tree used for assigning difficulty records to reported difficulties.

if a problem description explicitly referred to a moment in only one specified user's interaction (i.e., one moment of difficulty) this was recorded as one single id (and thus as one single difficulty record), with a reference to that specific user;

if a problem description referred to moments in all users' interactions, these were recorded as individual difficulty records for each individual user (so in case of a difficulty for all 8 users, this turned into 8 difficulty records);

if a problem description referred to moments in $\underline{n}$ specified users' interactions (where $n<$ the total number of users), the reported difficulties were recorded as $\underline{n \text { difficulty records each referring to an individual user; }}$

if a problem description referred to moments in $\underline{n}$ unspecified users' interactions (where $n<$ the total number of users), it was recorded as one single difficulty record, and a reference was made to ' $n$ users', without specifying the users (because they were unknown).

if a problem description referred to moments in a not specified number of unspecified users' interactions, it was recorded as one single difficulty record, stating 'unspecified' in the database field referring to users.

After ids were linked to the specific problem descriptions, a difficulty record was created for each id (figure 4, blocks in the middle). Figure 6 provides an overview of attributes of problem descriptions that were used as fields in the difficulty records. This part of the process resulted in a total of 466 difficulty records.

After entering as many attributes as possible for each of the moments of difficulty, usability problems were defined based on similarity. This was done in an iterative, data-driven process where the actual problem definitions emerged from the descriptions of the moments of difficulty themselves. Figure 7 shows some example usability problems. 
Figure 6. Attributes of moments of difficulty as specified in the fields of the difficulty records.

\begin{tabular}{|c|c|}
\hline Attributes (fields) & Possible values \\
\hline 1. ID & Unique number. \\
\hline 2. Lab that reported the problem & $<\mathrm{A}>,<\mathrm{B}>,<\mathrm{C}>$ \\
\hline 3. Participant & $\begin{array}{l}<\text { Name }>\text { and } \quad<\text { participant number }>\text {, or } \\
<\text { unspecified }>\text {, or }<\text { number of participants }>\end{array}$ \\
\hline 4. Data analysis approach & $<$ Lab specific $>$ or $<$ SlimDEVAN $>$ \\
\hline 5. Task & $\begin{array}{l}<\text { Task number }>\text { and short phrase indicating the task } \\
\text { (e.g., }<\text { Stop the cooking process }>\text { ) }\end{array}$ \\
\hline $\begin{array}{l}\text { 6. Description of difficulties and } \\
\text { causes assumed by the evaluator }\end{array}$ & Copied from the labs' reports in unedited form \\
\hline $\begin{array}{l}\text { 7. The action stage at which the } \\
\text { problem occurred }\end{array}$ & $\begin{array}{l}\text { Action stages based on Hartson }(2003):<\text { Planning }>\text {, } \\
<\text { Translation }>, \quad<\text { Physical }>, \quad<\text { Outcome }>, \\
<\text { Assessment }>,<\text { Independent }>\end{array}$ \\
\hline $\begin{array}{l}\text { 8. The context in which the problem } \\
\text { occurred. }\end{array}$ & $\begin{array}{l}\text { Exact reference in grammar-form to a screen image } \\
\text { on the product: Cooking_mode_setting (Tab: } \\
\text { Cooking, Menu: menu where mode for cooking can } \\
\text { be set) }\end{array}$ \\
\hline $\begin{array}{l}\text { 9. Target context (i.e., screen image } \\
\text { that would have been shown had the } \\
\text { participant performed the correct } \\
\text { action in the context of the provided } \\
\text { task) }\end{array}$ & $\begin{array}{l}\text { Exact reference in grammar-form to a screen image } \\
\text { on the product: Cooking_mode_setting (Tab: } \\
\text { Cooking, Menu: menu where mode for cooking can } \\
\text { be set) }\end{array}$ \\
\hline $\begin{array}{l}\text { 10. Action that would have been } \\
\text { correct in the context of the task and } \\
\text { would have led to the target context. }\end{array}$ & $\begin{array}{l}\text { Specified in loosely defined grammar that indicates } \\
\text { the required steps in detail. For example } \\
<\text { select_cooking_OK> (i.e., participant has to use } \\
\text { the rotary labeled 'select' to select the Cooking tab } \\
\text { and then press the button 'OK' to confirm the } \\
\text { selection). }\end{array}$ \\
\hline $\begin{array}{l}\text { 11. Action that was performed by the } \\
\text { participant }\end{array}$ & $\begin{array}{l}\text { Specified in loosely defined grammar that indicates } \\
\text { the steps performed by the participant in detail. For } \\
\text { example < press_select }>\text { (i.e., participant pressed the } \\
\text { rotary labeled 'select'). }\end{array}$ \\
\hline $\begin{array}{l}\text { 12. Relevance to the problem of a } \\
\text { number of high level issues } \\
\text { (variables). }\end{array}$ & $\begin{array}{l}\text { For each variable, relevance: }\langle\text { Yes }>,\langle\mathbf{N o}>\text {, } \\
<\text { Maybe }>\text {. Multiple variables can be relevant, at } \\
\text { least one is relevant. } \\
\text { Variables were: } \\
\text { - manually setting some cooking parameters } \\
\text { - making settings for selecting recipes } \\
\text { - actions for naming self-defined recipes } \\
\text { - actions for starting a cooking process, and for editing } \\
\text { or stopping an ongoing cooking process } \\
\text { - issues related to making general settings, like the } \\
\text { clock time etc. } \\
\text { - issues related to defrost settings } \\
\text { - issues related to low-level issues on how to use } \\
\text { buttons and menus } \\
\text { - issues related to the meaning and use of the 'Back' }\end{array}$ \\
\hline
\end{tabular}


Figure 7. Examples of usability problems.

Uses rotary to stop the cooking process
Presses rotary knob to stop the cooking process
Presses rotary knob to select a menu item or set
time
Participant hesitates to select EDIT for
prolonging the cooking time.
Inefficient having to do so many actions for
making settings: composing a name
Setting wintertime should not be done by just
changing the hours and minutes
Inconsistency between menu options with and
without default values
Misunderstanding that BACK in tabs menu
displays main menu showing the clock time.
Participant needs to be able to make longer names
for own recipes.

Moments of difficulty had to be similar in fairly high levels of detail for considering them to be the same problem. For example, one might argue that the first three problems mentioned in figure 7 essentially are instances of one underlying problem, namely a problem with the rotary knob. However, important in many comparative user studies and in studies conducted in design contexts are inferences about causes of reported problems. In this example case, the three mentioned problems led to different explanations of why the rotary-knob related problems occurred; in case of the first problem, participants in their attempts to stop the cooking process tried to set a very low temperature by turning the rotary, whereas in the second case participants most likely assumed that the cooking process could directly be stopped, by pressing the rotary knob. These problems refer to two different strategies for trying to stop the cooking process. As to the second and third problem: they both start from the same wrong use of the rotary knob (trying to press it, whilst this was not possible). In case of the second problem this was done with the aim of trying to immediately stop the cooking process, whereas in case of the third problem, this was done to select a menu item or to make a menu setting. Thus, these three problems are likely to have different causes and it is very likely that in a design context these problems would lead to different interface revisions. Identifying these three problems as one single problem might lead to false impressions of agreement similar to those reported by Hertzum, Jacobsen and Molich (2002). The process described above resulted in a list of 63 different usability problems (figure 4, block at the bottom). 
As the choice of the level of abstraction of problems is to some extent arbitrary and can be expected to influence agreement levels, a second way of categorizing problems was used as well. This other way of categorizing was purely based on the higher level issues as specified in the usability problem attributes (see figure 6, attribute 12). In this higher level categorization, problems were characterized by combining (mostly pairs of) higher level issues that were marked as relevant to the reported moment of difficulty. For example, if a moment of difficulty related to the issues manually setting the cooking parameters (abbreviated as Cooking) and low-level issues on how to use buttons and menu (in short Interaction techniques), it would be categorized as $<$ Cooking $>-<$ Interaction Techniques $>$. In cases where only one issue related to the moment of difficulty it was characterized as, for example $<$ Cooking $>-<$ Only $>$. In rare cases of more than two issues, all possible combinations of two marked issues were treated as separate categories. This resulted in a total of 35 high-level problem category pairs. For most analyses the (detailed) 63 problems were used. The 35 high-level problem category pairs were only used for comparing agreement measures.

\section{Findings on consistency and inspectability}

The process of creating a master list of usability problems proved to be hindered by how problem descriptions were structured (inconsistent formats), by the formulations that were used, as well as by the reports' lack of inspectability.

\section{Inconsistencies in problem formulations}

Sometimes problem descriptions were formulated in behavioral terms of difficulties encountered by users (e.g. appendix 4: "... users continued interacting thinking that the oven was still cooking" or "... one user tried to reduce the temperature... "). In other cases problem descriptions were formulated in terms of problematic product features; then it was sometimes unclear whether any of the users actually experienced the problem or what exact difficulty they had encountered (e.g., appendix 4: "Left hand arrow indicating that there is a submenu available is not clear"). In yet other cases, behavioral descriptions of problematic interaction episodes were given, but the difficulties themselves were not described separately. This sometimes made it difficult to infer what exactly the researchers thought the difficulty was or whether they thought that multiple moments of difficulty had occurred (e.g., appendix 1: "... Pressed rotary knob, turned. Presses 'stop' but puzzled when she saw...(etc.)").

The inconsistencies in problem descriptions hindered the construction of a master usability problem list. In cases where one of a lab's report described a 
problem in behavioral terms, and the other described it in terms of a problematic product feature, it often proved to be difficult to decide whether the descriptions actually referred to the same moment of difficulty; to be able to do so a comparer ${ }^{2}$ has to infer causal relationships between observed behavior and problematic product features. Similar problems occurred in cases where problematic interaction episodes were described without separate descriptions or marking of difficulties. In order to find out to what extent such a description refers to the same moment of difficulty as other problem descriptions formulated in terms of an encountered difficulty, a comparer has to infer what the analyst may have concluded about how many (and how many types of) difficulties are embedded in the described interaction.

\section{Useful information complementary to difficulty descriptions}

The core of the problem descriptions in the labs' reports (i.e., descriptions of difficulties and of problematic features) was often provided with complementary information. Such additional information sometimes proved to be essential (and often at least very helpful) for a better understanding of the problem. For example, mentioning the task in which a difficulty occurred provides context that can help in envisioning the situation in which the difficulty occurred. Suggestions for interface improvements or inferences about how design elements may have caused the problems may implicitly detail difficulty description (e.g., appendix 6: the description of the difficulty only states "... User presses rotary knob to stop the oven", whereas the inference about what may have caused the difficulty includes the statement: "... The rotary knob is the most dominant element among the controls, so that the user is directed to that without much intention." By stating this, the researcher implicitly details the difficulty description by suggesting that the user's focus of attention may have been at the wrong place.)

Thus, information complementary to the core of the problem description can be useful to a better understanding of the observed interaction. However, in a number of cases another problem then showed up. For example, in some cases suggestions for solutions or inferences about possible causes were not linked to specific observed difficulties on a one-to-one basis; instead, a group of inferences about causes were linked to a group of difficulties or reasons of difficulties. For example, in appendix 3, it is unclear whether the researcher intended to relate suggestion 3 about users' preferences for a stop/start button to one of three mentioned reasons or only to the main problem ("...Users find

\footnotetext{
${ }^{2}$ From now on the person who makes the comparisons between the lab reports will be referred to as the 'comparer' (for reasons of brevity).
} 
the stop-button easily, press the button, but are then confused by the feedback the oven provides").

Raw descriptions of what users said during or after interactions also provided complementary information that helped in better understanding ambiguous problem descriptions. For example in appendix 8: the main problem was formulated as "... When alarm is set it is not clear if time indicates time until alarm or indicates the actual time." The comment the user gives in the second finding at the bottom of the page "... I was not sure if duration was the total time or the time left" provides extra information that can be taken into account in trying to interpret the (more or less cryptic, main) problem formulation.

\section{Inspectability of data analyses}

In some lab reports, some of the raw descriptions referred to above were included and clarified reported problems. However, in many cases raw descriptions were missing and could only be found in the log overviews representing observed interactions. In order for that to be of any use, it should be possible to exactly trace back which raw descriptions relate to which problem. This relates to the issue of inspectability. Inspectability of data analyses can sometimes alleviate the problems mentioned above. For example, if the description of a problematic feature has some kind of reference to a specific moment that is captured in some representation of an interaction, that interaction may be re-inspected to find out what observation lies at the basis of the problem.

The primary reference needed for inspectability always is a reference to the specific user that encountered the difficulty. In cases where it is not specified which user encountered a difficulty, it becomes a very tedious and difficult job to go through all interactions and try and identify the exact session and moment at which the difficulty occurred. In those cases where this was tried, it usually ended up with various candidate moments from multiple users' interactions. No further information was then available for a better understanding of problem descriptions.

In addition to references to users, references to tasks also proved to be very helpful. Not only because the task description in itself helps in envisioning the context in which a difficulty might have occurred (see before), but also because it makes it possible to search in a more focused way for the specific interaction in which a difficulty has (or might have) occurred. In many cases such a reference to a task is not needed, because it is almost obvious in which task a difficulty must have occurred (e.g. appendix 4: it is very likely - though not certain - that the difficulty "... after the user presses 
Stop, there is no feedback that the oven has stopped cooking" refers to the task in which the user is asked to stop the cooking process). However, in some cases this is less clear (e.g., appendix 4: the problem left hand arrow indicating that there is a submenu available is not clear could have occurred in many tasks). In general, information about the task in which a difficulty occurred helped in finding back interaction episodes when overviews of interactions were available. Again, this was helpful because in such overviews complementary information could be found that helped in understanding the problem (e.g., verbal utterances of users, or for example, sequences of actions that helped re-constructing what the state of the product must have been at the time when the problem occurred).

\subsection{The comparisons}

The previous section described how problem descriptions were made comparable. This was done by first re-formatting them into uniformly structured difficulty records and by then constructing a master list of usability problems. This process of making findings comparable allowed for the comparisons that are described in the following section.

First, in section 4.2.1, comparisons between (findings from) the labs' initial and reference analyses will be discussed. Quantitative comparisons are made on the number of problems identified in each of the analyses, and the amount of overlap in problems was determined. An analysis is given on why certain problems were reported in one analysis and not in the other. This provides some information on consistency of analysts, when re-analyzing interactions. Experiences in trying to trace back causes of inconsistencies are then discussed and shed a light on inspectability of reports.

Next, in section 4.2.2, comparisons between labs are discussed, for the initial analyses as well as for the reference analyses. Quantitative comparisons are made of what was specified about each difficulty in the labs' problem descriptions, as well as about the extent to which labs reported similar or different problems (agreement or consistency across labs).

\section{Comparing problems reported in a lab's subsequent analyses}

For comparing how many problems the labs' subsequent analyses resulted in, a measure of thoroughness (Hartson, Andre and Williges, 2001) was used, and overlap in identified problems was examined. For calculating thoroughness and overlap, usability problems (from the master list of usability problems) were used as units of comparison. In addition, all problems that were uniquely identified either by a lab's initial analysis or by its reference analysis were further inspected to trace back reasons of uniqueness. For that inspection, usability problems were traced back to the difficulty records on 
which they were based and if necessary to the labs' original problem descriptions in their reports.

Thoroughness.

Hartson, Andre and Williges (2001) define thoroughness as the number of real problems found divided by the number of real problems that exist. In this case, it is assumed that all problems identified are real, as we have no reference criterion to determine whether problems are real or not. The number of real problems that exist, is defined here as the sum of all problems found by

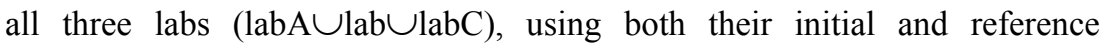
analyses $\left(\right.$ labX $X_{\text {labspecific }} \cup$ labX $\left.X_{\text {SlimDEVAN }}\right)$. Figure 8 presents the results of the calculations.

Figure 8. Thoroughness of data analyses (usability problems as unit of

\begin{tabular}{l|l|l}
\multicolumn{1}{c|}{ comparison) } & $\begin{array}{l}\text { Initial analyses } \\
(\text { lab-specific })\end{array}$ & $\begin{array}{l}\text { Reference analyses } \\
\text { (SlimDEVAN) }\end{array}$ \\
\hline $\mathrm{A} /(\mathrm{A} \cup \mathrm{B} \cup \mathrm{C})_{\text {labspecificuslimDEVAN }}$ & $26 / 63=41,3 \%$ & $29 / 63=46,0 \%$ \\
\hline $\mathrm{B} /(\mathrm{A} \cup \mathrm{B} \cup \mathrm{C})_{\text {labspecificuslimDEVAN }}$ & $23 / 63=36,5 \%^{3}$ & $27 / 63=42,9 \%$ \\
\hline $\mathrm{C} /(\mathrm{A} \cup \mathrm{B} \cup \mathrm{C})_{\text {labspecificuslimDEVAN }}$ & $23 / 63=36,5 \%$ & $33 / 63=52,4 \%$
\end{tabular}

For all labs, the second (reference) reports describe a larger number of problems than the initial lab reports. Thus the second analysis must have revealed problems that were not revealed in the initial analysis. However, based on the summative figures of thoroughness it cannot be excluded that the initial analysis also identified some unique problems. Thus, as a next step, it will be examined which analyses yielded unique problems and to what extent.

\section{Overlap and uniqueness}

Figure 9 shows that for all three labs, both the initial analyses as well as the reference analyses identified unique problems. Because the thoroughness of reference analyses was always higher than the thoroughness of initial analyses the number of unique problems was always higher for the reference analyses. Within-analyst consistencies (i.e., the proportion of overlap depicted in figure 9$)$ varied considerably across the three labs: $72 \%(=23 /(23+3+6))$ for lab A, $43 \%(=15 /(15+8+12))$ for lab B and $37 \%(=15 /(15+8+18))$ for lab C.

\footnotetext{
${ }^{3}$ Because for the reference analysis with SlimDEVAN the results of only six (instead of eight) participants were reported, the measures for the lab's initial report are based on the results of the same six participants.
} 
Figure 9. Number of problems identified in the labs' initial reports and in the labs' reference (second) reports. The black areas indicate overlap in problems between the first and second reports ${ }^{4}$.

\section{Initial report}

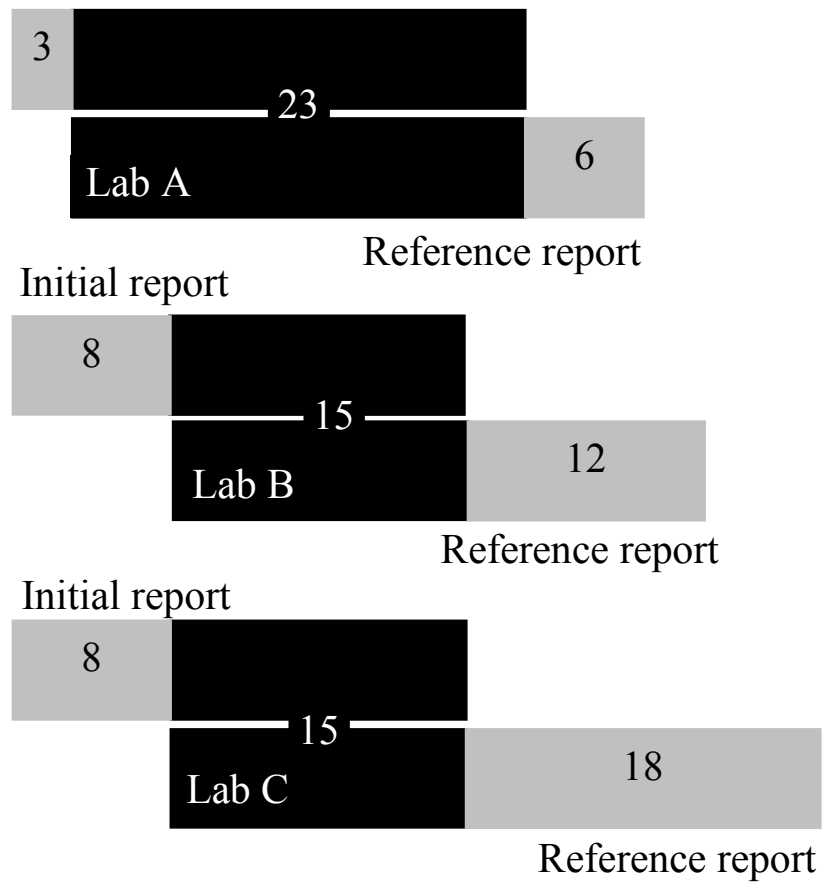

Inspecting why problems were reported in only one of the labs' analysis reports

Uniqueness of problems extracted in only one of the analyses can be explained in at least two ways. It can be due to methodological differences in the subsequent analyses (e.g., the focus of a data analysis procedure or the way usability problems are defined), or it can be due to something like 'chance' (because researchers, as part of the measurement instrument, can not be expected to be equally concentrated during all situations in their analysis).

\footnotetext{
${ }^{4}$ Because lab B's SlimDEVAN report reported results of only six (instead of eight) participants, the measures for the lab specific approach are based on the results of the same six participants.
} 
Below, unique problems are studied in more detail by trying to find out to what extent the specific moments of difficulty on which they were based had been observed and analyzed in both analyses, and how these were further interpreted in each of the analyses. For that, problems were first traced back to the difficulty records on which they were based and from there, if necessary and possible to available interaction overviews that provided detailed insight into what actually happened. For example, if a problem was identified in the reference analysis, but not in the initial analysis, one could search in the reports of the initial analysis to find out whether the moment of difficulty had been observed at all and had been interpreted differently, or whether it seemed to have been overlooked. Thus, if a moment of difficulty was uniquely found in the reference analysis and it was not possible to find anything back about it in the reports of the initial analysis, this tells something about the (un) inspectability of the report from the initial analysis; after all both analyses were based on the same observed interactions.

For 57 (out of 102) moments of difficulty that were uniquely based on one of the two reports it appeared to be impossible for the comparer to find anything about it in the other report because of inspectability problems. In the remaining 45 cases there were no inspectability problems and reasons of uniqueness could be analyzed. On closer inspection, in 10 out of these 45 cases a problem had incorrectly been considered unique; in these cases the comparer had problems in interpreting the problem report which then led to difficulties in recognizing the usability problem underlying the moment of difficulty. In 35 cases uniqueness could be traced back to specific parts of the data analysis process. Below these categories are dealt with in more detail.

\section{No inspection possible: unknown reasons of uniqueness}

Figure 10 shows how the 57 cases in which moments of difficulty appeared not be inspectable were distributed across the labs. There were two main reasons for hindering inspectability: (1) although the problems were described, the moments of difficulty on which they were based were not described; in such cases one has no starting points for inspecting the other report, or (2) it was not possible to link back from a problem description in the report to a moment of difficulty reported in the other report.

Figure 10 shows that 45 (out of the 57) moments of difficulty with uninspectable reason of uniqueness came from the reference reports (the sum of the values of the black bars), whereas 12 came from the initial reports (sum of white bar values). By analyzing the reasons of un-inspectability, it becomes clear to what extent these lie in the reference reports or in the initial reports. 
Figure 10. Moments of difficulty relating to unique problems for which it was not possible to inspect the cause of uniqueness.

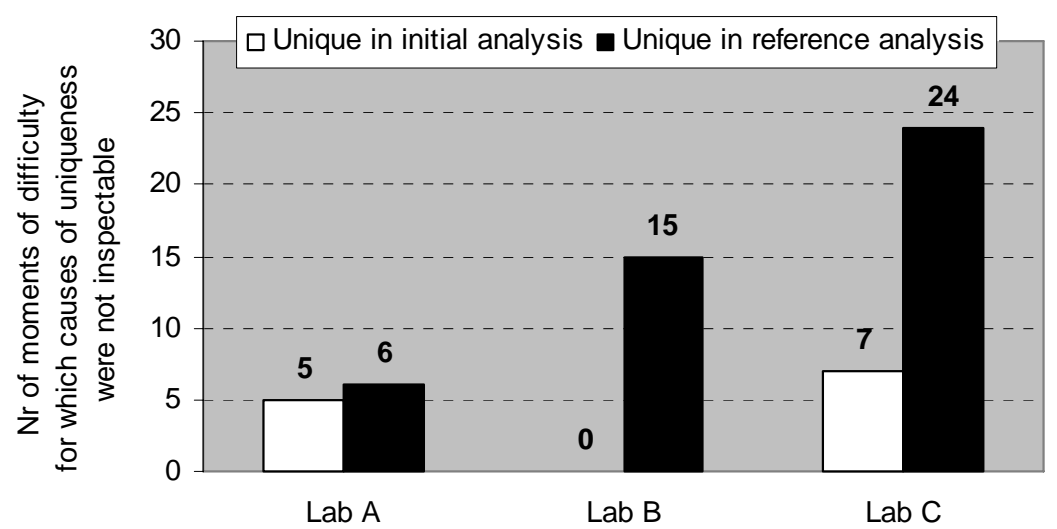

Lab A. In case of Lab A for five difficulties uniquely reported in the initial report it was not possible to find anything back in the reference reports. As no complete interaction overviews were available in the initial report (see appendix 1) the only way to inspect where the moment of difficulty is, would be to re-view the video tapes. Therefore, essentially this is a problem of inspectability of the initial reports that did not guide enough in where to search for the unique difficulty in the other analysis. In case of six moments of difficulty uniquely reported in the reference report, the reason of uniqueness was not traceable, again for the same reason: in the initial reports there were no complete interaction overviews for verifying whether the interaction that lies at the basis of the difficulty had even been observed or not.

Lab B. In case of Lab B for 15 moments of difficulty uniquely identified in the reference analysis, there was a lack of inspectability of the initial report. This lack of inspectability was a result of the fact that in the initial analyses (or at least in the initial report; see appendix 3) there were no (relatively complete) overviews that represented the users' interactions (there were only some example interactions embedded in the problem descriptions). Trying to inspect uniqueness of moments of difficulty would then imply re-viewing the video-recorded interactions for the specific task performance of the specific user again.

Lab C. In case of lab C there was a total of 24 moments of difficulty that were uniquely identified in the reference analysis, and for which there was an inspectability problem in relation to the initial report (see appendix 4). A major reason for that was that in the initial report those moments of difficulty 
had no reference to specific users, some also had no reference to a specific task and no interaction overviews were available. Therefore, it was practically impossible to reliably trace back the moments of difficulty identified in the reference analysis to something in the initial reports if the same problem descriptions were not explicitly mentioned there. Also the cases of the three unique moments of difficulty that were identified in the initial reports only were caused by the lack of inspectability of the initial reports. These concerned suggestions for solutions for which it was unclear whether any specific observed difficulty had been at the basis of it. In four other cases, the comparer had no idea why the problems had not been recorded in the reference analysis: a lack of interaction overviews in the initial reports made it impossible to verify whether the problem had actually occurred or not (unless the video recordings would be re-viewed).

Thus, in summary, the initial reports proved to be less inspectable than the reference reports. Problems of a lack of inspectability of the initial reports occurred largely because: initial reports provided no complete interaction overviews (lab A, B and C), no reference to specific tasks (lab C), no reference to specific users (lab $\mathrm{C}$ ) and because problems formulated in the initial report were written as suggestion with no apparent moment of difficulty mentioned (because of which it was unclear where to search in the reference reports or in available session logs of the initial reports, and the video would have to be re-viewed to see whether the difficulty actually occurred or not).

On closer inspection not unique: 'comparer' problems.

For ten (out of 102) moments of difficulty, the reason of uniqueness was caused by the fact that the comparer had had problems interpreting the problem report. In these cases, the inspectability of the reports made it possible to find out that the 'unique' moments of difficulty were not really unique. In eight of those cases the comparer had (on hindsight) made a wrong decision on whether a statement in a report should be interpreted as a problem or just as part of an overview of an interaction episode; this was the case for six records that were uniquely found in the reference analysis of lab A and for two unique moments of difficulty in the reference analysis of lab B. Here, problem descriptions were embedded in incomplete overviews of interaction episodes and were not separately and explicitly mentioned as such. In two other cases the comparer had interpreted a problem description in one analysis in a different way than he did in the other analysis. This happened with only one unique moment of difficulty from Lab B's initial analysis and with one unique moment of difficulty from lab C's reference analysis. In case of lab B this was due to an ambiguous problem formulation in the initial report, which was wrongly interpreted by the comparer.

Thus, to sum up, in some cases the comparer had made errors in interpreting problem descriptions. These errors related largely to ambiguous 
problem formulations (lab B) and lack of explicit distinctions between logs and problem descriptions (lab A and B) in the initial reports. This could only surface because in these cases the problem reports proved to be inspectable enough.

Unique problems: tracing back reasons of uniqueness.

In the analysis of the remaining 35 (out of 102) cases for which the comparer had concluded that a moment of difficulty was uniquely reported and for which the reasons were traceable, the following categories of inferred reasons for differences emerged:

(1) false-positives: moments of difficulty were reported but should not have been reported, because from the detailed analysis it has become clear that it is extremely unlikely that the problem actually occurred (and no other prove of existence was found other than the final problem description in the lab's report);

(2) slips in the analysis leading to missed moments of difficulty: the interaction in which the difficulty arose was observed in both analyses, but (for unknown reasons) was not recorded as a difficulty in one of the analyses even though it unambiguously represented a moment of difficulty;

(3) slips in the analysis leading to different problem descriptions: missed observations or utterances leading to different interpretations of moments of difficulty and thus to different interpretations of what exactly the problems were;

(4) threshold differences: differences in an analyst's decision of how difficult a difficulty should be for recording it as a moment of difficulty in the report;

(5) definition of 'usability problems': differences in what should lay at the basis of a usability problem (e.g., defining the fact that the user needed a hint as a moment of difficulty or not, distinction between problems of usability or other problems like software bugs).

Figure 11 shows that of the 35 unique moments of difficulty that were inspectable and regarded as being veritably unique, 23 were in the initial reports (sum of all values in the graph at the top of the figure) and 12 were in the reference reports (sum of all values in the graph at the bottom of the figure). 


\section{Figure 11. Reasons of uniqueness analyzed}
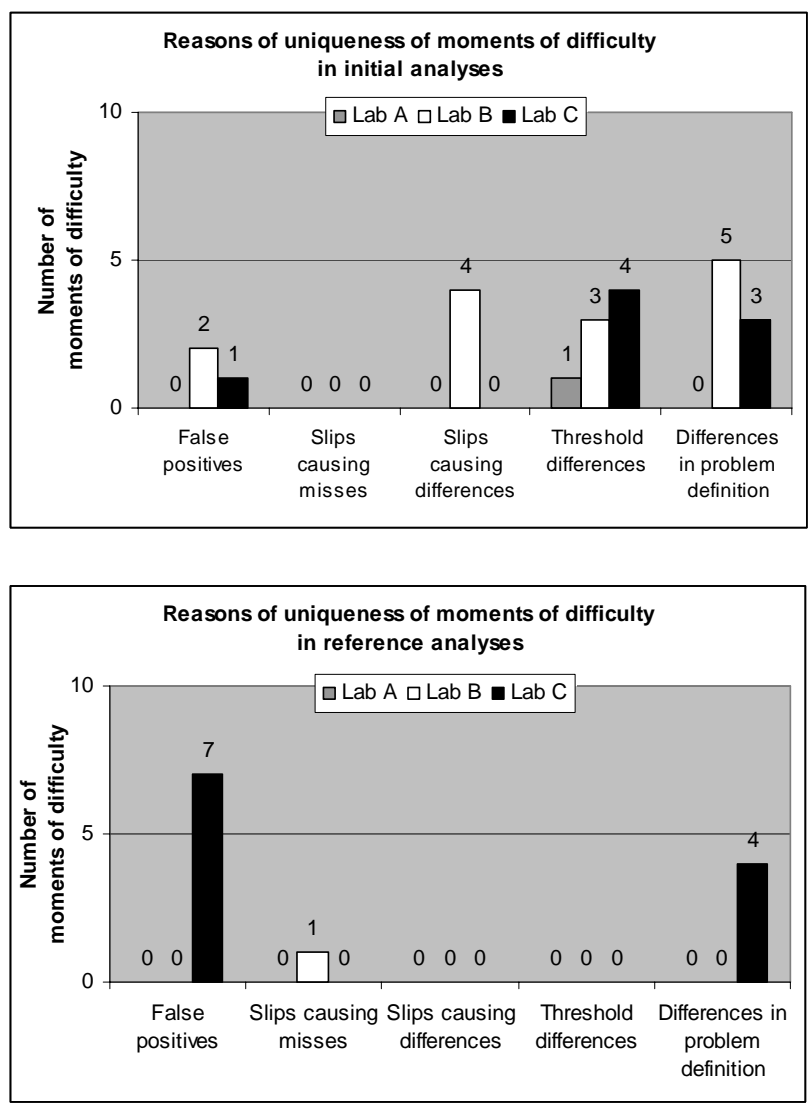

Lab A. In case of lab A (figure 11: grey bar), for the one moment of difficulty uniquely reported in the initial report that had been inspectable the reason of uniqueness was of type threshold differences. Based on the low number of inspectable moments of difficulty here, it may seem as if the reports of lab A had been relatively un-inspectable. However, one should bear in mind that lab A had the smallest number of unique moments of difficulty, among which a relatively large amount of comparer problems (which is a problem not of inspectability but of problem descriptions in the initial report).

Lab B. In case of lab B (figure 11: white bars), most 'real' unique moments of difficulty for which the reason had been inspectable were in the initial report (top graph of figure 11). Reasons of uniqueness were mainly of 
types: slips causing differences, threshold differences and definition of usability problems. In addition there were a few of types false-positives and slips causing misses. In case of the slips uniqueness usually had to do with not hearing or not noticing user's verbal utterances containing clues that helped in interpreting the specific moment of difficulty.

Lab C. In case of lab C (figure 11: black bars), reasons of uniqueness were found to be in the categories threshold differences and definition of usability problems. In addition, for eight moments of difficulty the reason of uniqueness was placed in the category false positives (seven of these were from the reference report).

Although the number of seven seems impressively large, it should be mentioned that these were seven instances of one single usability problem, and each of the instances was from a different user.

In summary, inspectability of the reports allowed for tracing back causes of uniqueness in these 35 cases. Reasons for uniqueness included: slips causing misses (lab B reference analysis), slips causing differences (due to for example missing user utterances) (lab B initial analyses), threshold differences (lab A, B, C), differences in definitions of usability problems (lab B and C) and false positives in the reference analysis (lab C).

\section{Comparing the reported problems across the three labs}

Some of the categories of reasons for uniqueness mentioned in the previous section seem to relate to issues of analysts working inaccurately (e.g., lack of attention), whereas others might be related to issues of differences in point of view, opinions or focus. For example, a more explicit and detailed procedure (as in the reference analysis with its checklist of signals of difficulty), may (but not necessarily does) bring about an implicit focus on specific elements of interactions or specific interpretations in formulating problems. If this would be the case, than one would expect to find less consistency between labs in the initial analysis (lab-specific analysis) than in the second (reference) analysis. Thus shifts in levels of agreement between labs when moving from the initial analyses to the subsequent analyses can tell something about the quality, in terms of biasing the results from the reference analysis. Below, levels of agreement across labs are calculated. First, comparisons are made of which problems were reported. Subsequently, comparisons are made on the type of information that is given about each problem. 


\section{Comparing which problems were reported}

For determining consistency in what problems each of the three labs reported, evaluator agreement measures are used. Hertzum and Jacobsen (2001) suggest the use of the any-two agreement measure in cases where the number of evaluators is relatively small. In this article, Hertzum and Jacobsen's (2001) definition is used, substituting evaluators by labs:

the average of $\frac{P_{i} \cap P_{j}}{P_{i} \cup P_{j}}$ (over all 1/2n(n-1) pairs of labs).

Similarly, agreement between $x$ labs can be defined as:

$$
\frac{P_{i} \cap P_{j} \cap \ldots \cap P_{x}}{P_{i} \cup P_{j} \cup \ldots \cup P_{x}}
$$

In these equations, $\mathrm{P}_{\mathrm{i}}, \mathrm{P}_{\mathrm{j}}$ and $\mathrm{P}_{\mathrm{x}}$ are the sets of problems (or high-level categories) detected by lab i, lab j, and lab x, and $\mathrm{n}$ is the number of labs.

\section{Figure 12. Agreement between labs (usability problems as unit of comparison)}

\begin{tabular}{l|l|l|} 
& Agreement between labs \\
\hline Usability problems & Lab specific & Slim-DEVAN \\
\hline Lab A vs. B & $11 / 38=28,9 \%^{5}$ & $11 / 45=24,4 \%$ \\
\hline Lab A vs. C & $13 / 36=36,1 \%$ & $15 / 47=31,9 \%$ \\
\hline Lab B vs. C & $10 / 36=27,8 \%$ & $16 / 44=36,4 \%$ \\
\hline $\begin{array}{l}\text { Any-two agreement } \\
\text { Three agreement Lab }\end{array}$ & $\mathbf{3 0 , 9 \%}$ & $\mathbf{3 0 , 9} \%$ \\
A-B-C & $(7 / 45=) 15,6 \%^{4}$ & $(9 / 56=) 16,1 \%$ \\
\hline
\end{tabular}

Figure 13. Agreement between labs (high-level problem categories as unit of comparison)

\begin{tabular}{l|l|l|}
\multicolumn{2}{|c|}{ of comparison) } \\
\hline $\begin{array}{l}\text { High-level problem } \\
\text { categories }\end{array}$ & Agreement between labs \\
& Lab specific & Slim-DEVAN \\
\hline Lab A vs. B & $12 / 23=52,2 \%$ & $15 / 28=53,6 \%$ \\
\hline Lab A vs. C & $12 / 23=52,2 \%$ & $14 / 23=60,9 \%$ \\
\hline Lab B vs. C & $13 / 19=68,4 \%$ & $17 / 31=54,8 \%$ \\
\hline Any-two agreement & $\mathbf{5 7 , 6 \%}$ & $\mathbf{5 6 , 4 \%}$ \\
\hline $\begin{array}{l}\text { Three agreement Lab } \\
\text { A-B-C }\end{array}$ & $(11 / 25=) 44,0 \%$ & $(13 / 31=) 41,9 \%$ \\
\hline
\end{tabular}

\footnotetext{
${ }^{5}$ Because in case of Lab B for the reference analysis (SlimDEVAN) the results of only six (instead of eight) participants were reported, the measures for the lab specific analysis of Lab B are based on the results of (the same) six participants as well.
} 
Figures 12 and 13 show the any-two agreement measures for usability problems and for high-level categories respectively.

Due to the higher level of abstraction (and hence the smaller number of categories) the higher any-two agreement for high level problem categories comes not unexpected. On both levels of abstraction, any-two agreement in the initial analysis is about the same as in the reference analyses. If the largely prescriptive analysis and reporting techniques of the reference analysis would have introduced a bias in finding usability problems, a higher level of agreement would have been expected for the second (reference) analyses. No indications were found that the reference analysis leads to a specific bias in the labs' results. In other words, the level of agreement does not seem to be influenced by the fact that the labs' analysis methods used in the initial analyses were different, whereas those in the second analyses were similar.

\section{Comparing 'what was specified about each problem'}

Figure 14 shows, for each of the analyses, an overview of the elements that problem descriptions consisted of in case of each of the three labs. For that, the fields difficulty and cause in each difficulty record (see figure $6,6^{\text {th }}$ attribute) were analyzed. The contents of these difficulty record fields had been taken literally from the labs' reports. These difficulty records were analyzed to find out to what extent they mentioned (or otherwise referred to) the following problem description elements:

(1) the situation in which the problem occurred (was the product status mentioned, was there a mentioning of preconditions for actions that were not met?)

(2) the user's observable behavior at the time the difficulty occurred (what parameters was the user trying to set, what physical action was the user trying to perform?)

(3) what the user thought, felt or understood (explicit mentioning of inferences about the user's reasoning, understanding, feelings or about what the user tried to achieve)

(4) what the effect of the difficulty was (this relates to the effect of the problem on the product status, as well as effects on the user, like confusion, frustration, etc.)

(5) inferences about what product element had caused the difficulty or should be redesigned to avoid it (explicit mentioning of product characteristics that are believed to have contributed to the difficulty, of the reason why they are believed to have contributed to it, as well as suggestions to change the functionality of the product).

Compared are the proportions of problem descriptions that contained statements referring to each of the mentioned descriptive elements. Thus, the figures are corrected for differences in absolute numbers between the initial analyses and the reference analyses. 
Figure 14. Overview of what descriptive elements the labs used in their problem descriptions.
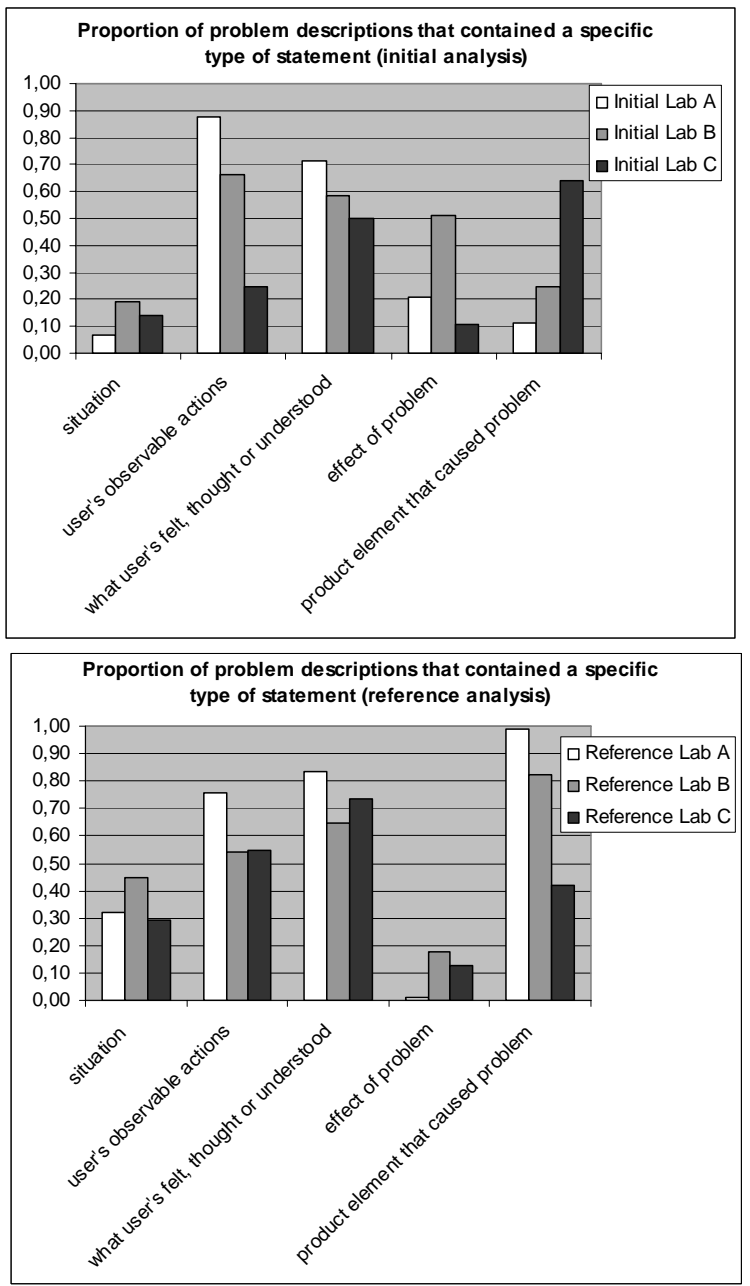

Figure 14 shows that in the graph at the bottom, the relations between the bars per lab (i.e., all bars of a certain color) are more similar than in the graph at the top. In other words, it seems that in the second analysis labs have produced more similar reports in terms of what they reported about problems.

Summarizing, the reference analysis procedure did not lead to more agreement on what usability problems were extracted, but it did lead to more consistency in what was reported about each of the problems. Labs reported more about the situation in which problems occurred and were more in 
agreement with each other with respect to the amount of problem descriptions containing behavioral observations and inferences about what users seemed to understand, feel and think. Especially lab $\mathrm{C}$, which originally reported relatively little about what users did, now reported more about that. The labs that in the initial reports mentioned only few possible causes of problems (labs A and B), now showed a considerable increase, whereas lab C that already was at a high level, reported relatively less causes. Finally, for labs A and B, the reporting of effects of actions dropped to a very low level in the reference analysis, whereas lab C stayed at an already relatively low level.

\section{DISCUSSION AND CONCLUSION}

The aim of the study was to investigate issues of consistency and inspectability of data analyses and reports based on usability tests meant to extract usability problems. Consistency of findings was studied for analysts who analyzed their observed interactions twice with time intervals of one to two months (the first time using their own usual method of analysis, the second time all using the same reference method of analysis). Inconsistencies were analyzed in detail in order to trace back their origin. Consistency across labs was studied in terms of levels of agreement on problems and high-level categories; it was determined to what extent these levels change when the labs, in their re-analysis, all use the same data analysis method. Also, it was explored to what extent the use of the same data analysis approach led to the use of similar descriptive elements in the labs' problem descriptions. By making the reported usability problems comparable as well as by analyzing reasons of inconsistencies, it became clear which problem report elements were important for (1) making reports inspectable and (2) making the reported findings traceable to the original observations. Below the findings on inspectability and consistency are discussed.

\subsection{Inspectability}

Inspectability of usability problem reports is important for laying bare those elements in a data analysis process that involve subjective analyst judgments. In this study it became clear that for comparative studies inspectability is also important for two other reasons. Firstly, it proved to be of crucial importance for understanding reported problems in enough detail to be able to decide on their similarity. Secondly, inspectability proved to be of major importance for tracing back reasons of why a lab reported a problem in one analysis and not in the other. For example, problems of inspectability when trying to trace back the origin of uniqueness were present in 57 of the 102 problem records that were reported in just one of a lab's analyses. 
The absence of the following report characteristics proved to be a hindrance in tracing back findings to more primary data. In other words, to make usability problem reports more inspectable, the following issues can be helpful:

(1) inclusion of descriptions of a user's actual behavior in the description of a problem, e.g., descriptions of problematic user actions;

(2) making references to which users encountered a problem;

(3) making references to the task context or the (sub) goal that a user was trying to accomplish at the time of the problem;

(4) making clear distinctions between descriptions of problematic interactions and descriptions of interactions that are interpreted as proceeding without problems;

(5) inclusion of (or making references to) overviews of observed interactions (without substantial gaps), instead of only relying on problem descriptions;

(6) inclusion of an account of a user's verbal utterances, as well as clear links between such account and interaction overviews or problem descriptions;

(7) inclusion of inferences about causes of problems (or suggestions for how to resolve problems) with clear links to behavioral problem descriptions.

\subsection{Consistency}

\section{Across-analyst consistency (subjectivity)}

Comparison of what descriptive elements were used to report problems showed that the (SlimDEVAN) reference reports led to more across-lab consistency, than the initial reports. In other words, without using an agreed method for reporting problems, consistency across analysts on what elements are used to report problems may suffer; this in turn makes it more difficult to compare the usability problems identified by those analysts. In the present study, the SlimDEVAN method led to more consistency in the degree to which the analysts provided descriptions of the situations in which problems occurred, of the behavioral descriptions of observable problematic actions, of accounts indicating what analysts inferred users were feeling, understanding or thinking, and in mentioning product elements that presumably had caused problems.

Although consistency in what descriptive elements were used to report problems was higher in the reference reports than in the initial reports, measures of consistency on which problems were identified tell a different story: any-two agreement levels were about the same for the initial and reference analyses. This implies that adopting similar data analysis approaches and using an agreed format for describing usability problems not necessarily 
leads to more consistency in findings across analysts. Any-two agreement of the labs (comparing usability problems) was 30,9\% for both analyses. Based on our data, it is unclear whether the lack of agreement stems (1) from differences in the labs' original data (e.g., actual differences in interactions, because the labs used different test participants in different countries) or (2) from some analyst-specific (i.e.,: not method-specific) characteristic of the analysis (e.g., inherent subjectivity in the data analysis process).

\section{Within-analyst consistency}

For measuring within-analyst consistency two measures were used: (1) thoroughness of the first analysis and of the second analysis; (2) overlap: the number of problems that a lab found in both analyses, divided by the total number of problems they found in the two analyses.

In the reference analysis (the second analysis) thoroughness proved to be consistently higher than in the initial analysis. Two possible reasons are: (1) problems identified in the initial analysis may have a higher chance of being identified again in the second analysis, while at the same time analysts have a second chance for identifying problems that were overlooked in the first analysis; (2) due to its characteristics the reference method is able to identify a larger number of problems. The present data do not allow determining to what extent these reasons contribute to the current findings.

The proportion of overlap of findings from the initial and subsequent analyses was found to be substantially different across labs. One can think of two possible reasons: (1) differences in levels of thoroughness between initial and subsequent analyses varied considerably (e.g., more thoroughness in the analyses increases the chance for more overlap); (2) the methods the labs used in their initial analyses differed in the extent to which they facilitate analysts to work in a consistent manner. Based on the available data it is unclear which of these reasons is most likely.

The reasons behind the findings on (within-analyst as well as acrossanalyst) consistency all raised some questions. Due to issues of confounding in the set up of the study, the questions can not be answered just by using the data of the present study. In making within-analyst comparisons there was confounding due to the analysis methods the labs used as well due to an order effect (labs used different methods in their first and second analysis); in the across-analyst comparisons, there was confounding due to the fact that the three labs worked from observations of different test participants. Data from a separate study described in Vermeeren, Koenderink-van Doorn and de Ridder (2006) suggest possible answers. These are dealt with below. 


\subsection{Follow-up study on causes of (in)consistency}

In the study described in Vermeeren, Koenderink - van Doorn and de Ridder (2006) two pairs of students (in the context of a course on research methodology) were asked to analyze parts of the recordings from lab B twice with an interval of about three weeks and to use SlimDEVAN for both their analyses. Students were free to decide how many and which sessions they analyzed. They were third-year (Bachelor) students of Industrial Design Engineering at Delft University of Technology and had no experience in formal user testing and data analysis. However, they had three years of experience in practical courses on user-centered design and evaluation of consumer products. Students were provided with the English language user manual of SlimDEVAN (Vermeeren, 2003), with an abbreviated Dutch language user manual as well as with published literature on DEVAN (Vermeeren, van Kesteren \& Bekker, 2003; Vermeeren, 2004). In addition, the first author of the present article explained and discussed SlimDEVAN with each individual pair of analysts. In addition, after they had performed their first session, analysts had their analysis checked by the first author of the present paper to identify misunderstandings of SlimDEVAN.

Students analyzed the sessions of their choice twice with a minimum interval of three weeks. During those weeks the students did not watch the recorded sessions nor did they review their analyses; largely they spent time on doing exams for other courses as well as on doing a literature search on the topic of comparing usability evaluation methods. Student pair 1 decided to analyze the sessions of test participants 1,2,3, 4 and 5; student pair 2 analyzed the sessions of test participants $2,4,5$ and 6 .

The following measures were taken to study issues of consistency:

- across-analyst consistency in the second analysis, in terms of agreement between findings of each student pair and of lab B (expressed as a proportion of the total number of problems identified by the lab and the student pair in their second analysis). Figures are based on the same sample of test participants for student pair and lab B). See figure 15 for the results.

- thoroughness of the students' first and second analyses in comparison to thoroughness of Lab B's analyses (again for the same sample of test participants). Total number of usability problems that exist is defined here as the total number of problems identified by all three labs and the two student pairs in their first and second analyses ( 77 problems). See figure 16 for the results.

- within-analyst consistency in terms of overlap between first and second analysis in comparison to that of lab B (for the same sample of participants). Overlap is expressed as the proportion of the total number 
of problems identified by a lab/student pair in their two analyses. See figure 17 for the results.

Figure 15. Analyst agreements of student pairs and lab $B$ in the second analysis (proportion of the total number of problems identified by the lab and the student pair in their second analysis).

\begin{tabular}{lll}
\hline $\begin{array}{l}\text { Analyst } \\
\text { combination }\end{array}$ & $\begin{array}{l}\text { Participant } \\
\text { sessions }\end{array}$ & $\begin{array}{l}\text { Analyst agreement } \\
\text { second analysis } \\
\text { (SlimDEVAN) }\end{array}$ \\
\hline $\begin{array}{l}\text { Student pair 1 } \\
\text { vs. Lab B }\end{array}$ & $1,2,3,4,5$ & $26,8 \%$ \\
\hline $\begin{array}{l}\text { Student pair 2 vs. } \\
\text { Lab B }\end{array}$ & $2,4,5,6$ & $29,4 \%$ \\
\hline
\end{tabular}

In the discussion, the question was raised as to what had caused the limited agreement in the labs' findings: would it be due to differences in the observed interactions themselves (as the labs had used different participants) or to issues of analyst subjectivity. Even though each of the student pairs and lab B had analyzed exactly the same data (i.e., the same sessions of the same participants), the levels of agreement still are roughly in the same range as those of the labs (i.e., $24,4 \%, 31,9 \%$ and $36,4 \%$ for the SlimDEVAN analyses; see figure 12). This is contrary to the expectation one would have if the difference in original data would have been the most important factor for the lack of agreement. This suggests that analyst-specific factors (i.e., subjectivity) play an important role in the lack of agreement. The fact that agreement levels between lab B and the student groups were even lower than agreement levels between labs may be explained by the fact that these were based on data from a smaller number of participants.

Figure 16. Thoroughness of analyst groups compared across $1^{\text {st }}$ and $2^{\text {nd }}$ analysis. Total number of usability problems that exist is $\mathbf{7 7}$.

\begin{tabular}{|c|c|c|c|c|}
\hline Analysts & $\begin{array}{l}\text { Participant } \\
\text { sessions }\end{array}$ & $\begin{array}{c}\text { Thoroughness } \\
\text { 1st analysis }\end{array}$ & $\begin{array}{l}\text { Thoroughness } \\
\text { 2nd analysis }\end{array}$ & $\begin{array}{c}\text { Increase in } \\
\text { thoroughnes } \\
\mathrm{s} \\
\end{array}$ \\
\hline $\begin{array}{l}\text { Student } \\
\text { pair 1 }\end{array}$ & $1,2,3,4,5$ & $\begin{array}{c}31,2 \% \\
\text { (SlimDEVAN) }\end{array}$ & $\begin{array}{c}32,5 \% \\
\text { (SlimDEVAN) }\end{array}$ & $1,3 \%$ \\
\hline Lab B & $1,2,3,4,5$ & $\begin{array}{c}29,9 \% \\
\text { (Lab specific) }\end{array}$ & $\begin{array}{c}35,1 \% \\
\text { (SlimDEVAN) }\end{array}$ & $5,2 \%$ \\
\hline $\begin{array}{l}\text { Student } \\
\text { pair } 2\end{array}$ & $2,4,5,6$ & $\begin{array}{c}49,4 \% \\
\text { (SlimDEVAN) }\end{array}$ & $\begin{array}{c}53,3 \% \\
\text { (SlimDEVAN) }\end{array}$ & $3,9 \%$ \\
\hline Lab B & $2,4,5,6$ & $\begin{array}{c}26,0 \% \\
\text { (Lab-specific) }\end{array}$ & $\begin{array}{c}32,5 \% \\
\text { (SlimDEVAN) }\end{array}$ & $6,5 \%$ \\
\hline
\end{tabular}


With respect to the observed increase of thoroughness from the first to the second analyses, the question arose: to what extent would this be due to the fact that the second analysis is a re-analysis or to some method-specific factor. In figure 16, the observed increase in thoroughness for the student pairs (i.e., $1,3 \%$ and $3,9 \%$ ) can only be due to the sole fact that the second analysis was a re-analysis. A similar increase in thoroughness for lab B would suggest the same underlying reason. However, figure 16 shows that the increase for lab B is higher. This suggests that the increase in the labs' thoroughness is partly due to the fact that the analysis is a re-analysis (i.e., in the range of 1,3 - 3,9\%) and partly due to differences between the methods that were used in the first and second analysis (i.e., one of the methods being more thorough than the other). Further research is needed to substantiate these speculations.

Figure 17. Within-analyst consistencies of analyst groups and lab B compared (proportion of the total number of problems identified by a lab/student pair in their two analyses).

\begin{tabular}{llclc}
\hline Analysts & $\begin{array}{l}\text { Participant } \\
\text { sessions }\end{array}$ & $1^{\text {st }}$ Analysis & $2^{\text {nd }}$ Analysis & $\begin{array}{c}\text { Within- } \\
\text { analyst } \\
\text { consistency }\end{array}$ \\
\hline Student pair 1 & $1,2,3,4,5$ & SlimDEVAN & SlimDEVAN & $63,3 \%$ \\
Lab B & $1,2,3,4,5$ & Lab specific & SlimDEVAN & $42,9 \%$ \\
\hline Student pair 2 & $2,4,5,6$ & SlimDEVAN & SlimDEVAN & $71,7 \%$ \\
Lab B & $2,4,5,6$ & Lab specific & SlimDEVAN & $36,4 \%$ \\
\hline
\end{tabular}

The question regarding within-analyst consistency in terms of overlap was whether this would relate to thoroughness or to differences in methods (as some methods may make it easier to work in a consistent manner than others). The findings in figures 16 and 17 suggest no clear relation between thoroughness and within-analyst consistency; the highest and lowest withinanalyst consistency are found for the analyses with the highest and lowest thoroughness, but within-analyst consistency of student pair 1 is also high without their thoroughness being quite high. Note that within-analyst consistency is high for both student pairs and low for lab B. This suggests that indeed the used analysis method plays an important role in analysts being consistent across subsequent analyses.

\section{Conclusions}

The findings in the case study together with those from the complementary study suggest that:

(1) the levels of agreement between analysts relate to analyst-specific characteristics, rather than to characteristics of the used analysis methods or to differences in the original data. In other words there seems to be considerable inherent subjectivity in findings from a usability test based 
on identifying problems and consistency across analysts is not very likely to be improved by using specific analysis methods;

(2) re-analysis of the same data is very likely to lead to a slightly higher thoroughness in the second analysis even if the second analysis is done one or two months later (in this case in the range of 1-4\%);

(3) within-analyst consistency does seem to be sensitive to the used analysis method; using the SlimDEVAN method in subsequent analyses led to within-analyst consistencies of $63-72 \%$, whereas within-analyst consistencies of labs using different methods in their first and second analyses were, on average $50 \%$.

\section{Implications}

In usability studies that are based on comparing problems, it is important that all data are analyzed by the same analysts. This is important because of the inherent subjectivity in extracting usability problems from observations. With appropriate methods that conform to the characteristics as present in SlimDEVAN and as mentioned in section 5.1, within-analyst consistency can be improved. Also, such methods make the findings inspectable. Inspectability is important for laying bare subjective parts in data analyses and for making sure that outside reviewers can falsify or confirm the findings. In comparative usability studies inspectability is also important for a thorough understanding of the reported problems. This is crucial for being able to decide on similarity of problems.

\section{REFERENCES}

Barendregt, W., \& Bekker, M.M. (2005). Developing a coding scheme for detecting usability and fun problems in computer games for young children. Proceedings of Measuring Behaviour 2005, Wageningen, The Netherlands.

Cockton G., Lavery D. \& Woolrych A. (2002). Inspection-based evaluations. In J. Jacko \& A. Sears (Eds.) The human-computer interaction handbook: fundamentals, evolving technologies and emerging applications, (pp. 1118 - 1138) Mahwah, NJ: Lawrence Erlbaum Associates.

Daams, B. \& Hariandja J. (2006). Cross-cultural usability, testing a food processor in Indonesia and the Netherlands. Proceedings of IEA2006: 16th World Congress on Ergonomics IEA2006 , 1-5, Amsterdam, The Netherlands: Elsevier. 
Guba E.G., \& Lincoln Y.S., (1989). Fourth generation evaluation. London: Sage.

Hartson, H.R. (2003). Cognitive, physical, sensory and functional affordances in interaction design. Behaviour \& Information Technology, 22 (5), 315-338.

Hartson, H.R., Andre, T.S., \& Williges, R.C. (2001). Criteria For Evaluating Usability Evaluation Methods. International Journal of HumanComputer Interaction 13 (4), 373-410.

Hertzum, M. \& Jacobsen N.E. (2001). The Evaluator Effect: A Chilling Fact About Usability Evaluation Methods. International Journal of Human-Computer Interaction, 13(4), 421-443.

Hertzum, M., Jacobsen, N.E. \& Molich, R. (2002). Usability Inspections by Groups of Specialists: Perceived Agreement in Spite of Disparate Observations. Extended abstracts of the ACM CHI 2002 Conference, 662-663, New York: ACM.

Hoenderdos, R., Vermeeren, A.P.O.S., Bekker, M.M. \& Pierik A. (2002)

Design for experience: the "Look, mama!" experience. Proceedings of Interaction Design and Children, 4-10, Eindhoven: The Netherlands.

Jacobsen, N.E., Hertzum, M. \& John, B.E. (1998). The Evaluator Effect in Usability Tests. Proceedings of the CHI'98 Conference on Human Factors in Computing Systems (Summary), 255-256, New York: ACM.

Kanis, H. (1993). Reliability in Ergonomics/Human Factors. Contemporary Ergonomics, 91-96, London: Taylor and Francis.

Lavery, D., Cockton, G., \& Atkinson M. (1997). Comparison of evaluation methods using structured usability reports. Behaviour \& Information Technology, 16 (4), 246-266.

Law E. L.-C. \& Hvannberg E.T. (2004). Analysis of Combinatorial User Effect in International Usability Tests. Proceedings of ACM CHI 2004 Conference on Human Factors in Computing Systems. April 24-29, 2004, 9-16, Vienna, Austra. 
Molich, R., Ede M.R., Kaasgaard K. \& Karyukin B. (2004). Comparative usability evaluation. Behaviour \& Information Technology, 23 (1), 65-74.

Noiwana J., \& Norcio A.F. (2006). Cultural differences on attention and perceived usability: Investigating color combinations of animated graphics. International Journal of Human-Computer Studies 64 (2), $103-122$.

Park K.S. \& Lim C.H. (1999). A structured methodology for comparative evaluation of user interface designs using usability criteria and measures. International Journal of Industrial Ergonomics 23, 379 389.

Vermeeren, A.P.O.S., (1999). Designing scenarios and tasks for user trials for home electronic devices. In W.S. Green \& P.W. Jordan (Eds.), Human Factors in Product Design: current practice and future trends (pp 47-55). London: Taylor \& Francis.

Vermeeren, A.P.O.S. (2003). The DEVAN checklist for detecting problems in interactions: user manual (checklist version 1.1) (Technical Report). Delft, The Netherlands: Delft University of Technology, Faculty of Industrial Design Engineering.

Vermeeren, A.P.O.S. (2004). Structured Video Analysis of User test Data, using the DEVAN Tool. In Ç. Erbuğ (Ed.) Usability testing: Methods, Experiences, Achievements (pp. 123-140). Ankara, Turkey: Middle East Technical University, Faculty of Architecture,.

Vermeeren, A.P.O.S., Bekker, M.M., van Kesteren, I.E.H. \& de Ridder, H., (2007). Experiences with Structured Interviewing of Children during Usability Tests. In L.J. Ball et al. (eds.) Proceedings of HCI 2007, The $21^{\text {st }}$ British HCI Group Annual Conference, 139-146. Swindon, UK: BCS.

Vermeeren, A.P.O.S., den Bouwmeester, K., Aasman, J., \& de Ridder, H. (2002). DEVAN: a tool for detailed video analysis of user test data. Behaviour \& Information Technology, 21 (6), 403-423.

Vermeeren, A.P.O.S., van Kesteren, I.E.H., \& Bekker M.M. (2003). Managing the Evaluator Effect in User Testing. Proceedings of INTERACT'03, 647-654, Amsterdam, The Netherlands: IOS Press. 
Vermeeren A.P.O.S., Koenderink-van Doorn A.J., Ridder H. de (2006)

Reliability of a check-based user test data analysis procedure.

Proceedings IEA 2006 Congress: Meeting Diversity in Ergonomics, Maastricht, The Netherlands.

\section{ACKNOWLEDGMENTS}

Many thanks to the anonymous reviewers and to review editor Clayton Lewis for their well-considered and helpful comments and suggestions on an early draft of this article. Also, the authors would like to thank the test participants for their efforts in the usability tests, Deana McDonagh, Pelin Gültekin and Zeynep Karapars for their contribution to the user tests, Nigel Bevan for his contributions in the early phases of the collaborative project and Jans Aasman for comments on an early draft of this article. 
Appendix 1. Example of part of a session log from lab A (lab specific analysis; task: stop cooking). In the top row, characteristics of the participant are given. The various columns show (1) the task, (2) task effectiveness (either 1 or 0 ), (3) free-form problem description/logged actions, (4) evaluator's suggestions for solutions to the problem, (5) general comments, (6) answer to the question whether the product met the participant's expectations with respect to this task.

\begin{tabular}{|c|c|c|c|c|c|}
\hline$<$ Name $>$ & 41 & High & Female & & \\
\hline Task & Effectiveness & Problem & Suggestion & Comments & $\begin{array}{l}\text { Meets } \\
\text { expectations? }\end{array}$ \\
\hline Stop & 1 & $\begin{array}{l}\text { Pressed rotary knob, } \\
\text { turned. Pressed } \\
\text { 'stop' but puzzled } \\
\text { when she saw 'menu' } \\
\text { and 'cooking'. } \\
\text { Pressed 'stop' } \\
\text { unintentionally. }\end{array}$ & $\begin{array}{l}\text { Display may } \\
\text { turn dark; } \\
\text { feedback or } \\
\text { a text may } \\
\text { appear on } \\
\text { the screen }\end{array}$ & $\begin{array}{l}\text { Not safe, } \\
\text { Didn't } \\
\text { provide } \\
\text { feedback }\end{array}$ & \\
\hline
\end{tabular}


Appendix 2. Example of a part of lab B's session logs (lab specific analysis). Columns show (from left to right): date and time; recorded events; code for participant; task; video frame number; reference to video file.

\begin{tabular}{|c|c|c|c|c|c|}
\hline & $\begin{array}{l}\text { [SYS][switched to task Prolong } \\
\text { the cooking time] }\end{array}$ & 36 & $\begin{array}{l}\text { Prolong the } \\
\text { cooking time }\end{array}$ & 1589 & \\
\hline $\begin{array}{r}28-10-2003 \\
10: 35\end{array}$ & start & 36 & $\begin{array}{l}\text { Prolong the } \\
\text { cooking time }\end{array}$ & 1591 & $\begin{array}{l}\text { NL281003_930_riette.av } \\
\mathrm{i}\end{array}$ \\
\hline $\begin{array}{r}28-10-2003 \\
10: 35\end{array}$ & $\begin{array}{l}\text { I will make it thiryt minutes ... iot his } \\
\text { right }\end{array}$ & 36 & $\begin{array}{l}\text { Prolong the } \\
\text { cooking time }\end{array}$ & 1593 & $\begin{array}{l}\text { NL281003_930_riette.av } \\
\mathrm{i}\end{array}$ \\
\hline $\begin{array}{r}28-10-2003 \\
10: 35\end{array}$ & $\begin{array}{l}\text { I forgot to check how many } \\
\text { minutes you still have to go... what } \\
\text { is twenty minutes more }\end{array}$ & 36 & $\begin{array}{l}\text { Prolong the } \\
\text { cooking time }\end{array}$ & 1602 & $\begin{array}{l}\text { NL281003_930_riette.av } \\
\mathrm{i}\end{array}$ \\
\hline $\begin{array}{r}28-10-2003 \\
10: 36\end{array}$ & $\begin{array}{l}\text {... Im not sure I forgot to check how } \\
\text { many minuytes to go... }\end{array}$ & 36 & $\begin{array}{l}\text { Prolong the } \\
\text { cooking time }\end{array}$ & 1624 & 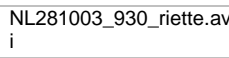 \\
\hline $\begin{array}{r}28-10-2003 \\
10: 36\end{array}$ & stops and explains & 36 & $\begin{array}{l}\text { Prolong the } \\
\text { cooking time }\end{array}$ & 1650 & $\begin{array}{l}\text { NL281003_930_riette.av } \\
\mathrm{i}\end{array}$ \\
\hline $\begin{array}{r}28-10-2003 \\
10: 36\end{array}$ & you did not check the temperaty & 36 & $\begin{array}{l}\text { Prolong the } \\
\text { cooking time }\end{array}$ & 1670 & $\begin{array}{l}\text { NL281003_930_riette.av } \\
\mathrm{i}\end{array}$ \\
\hline $\begin{array}{r}28-10-2003 \\
10: 36\end{array}$ & I will try & 36 & $\begin{array}{l}\text { Prolong the } \\
\text { cooking time }\end{array}$ & 1676 & $\begin{array}{l}\text { NL281003_930_riette.av } \\
\mathrm{i}\end{array}$ \\
\hline $\begin{array}{r}28-10-2003 \\
10: 36\end{array}$ & goes to edit & 36 & $\begin{array}{l}\text { Prolong the } \\
\text { cooking time }\end{array}$ & 1679 & $\begin{array}{l}\text { NL281003_930_riette.av } \\
\mathrm{i}\end{array}$ \\
\hline $\begin{array}{r}28-10-2003 \\
10: 37\end{array}$ & goes to temperatue & 36 & $\begin{array}{l}\text { Prolong the } \\
\text { cooking time }\end{array}$ & 1683 & $\begin{array}{l}\text { NL281003_930_riette.av } \\
\mathrm{i}\end{array}$ \\
\hline $\begin{array}{r}28-10-2003 \\
10: 37\end{array}$ & change temperature & 36 & $\begin{array}{l}\text { Prolong the } \\
\text { cooking time }\end{array}$ & 1687 & $\begin{array}{l}\text { NL281003_930_riette.av } \\
\mathrm{i}\end{array}$ \\
\hline
\end{tabular}




\section{Appendix 3. Example of how lab B reported its findings (lab specific analysis).}

\section{Task 1 Stop the oven}

\section{Problems related to task}

\section{Find the stop button:}

Users find the stop-button easily, press the button, but are then confused by the feedback the oven provides.

Reasons:

1. Display still says "cooking", indicating a menu-item. Novice users interpret it as feedback: the oven is still cooking.

2. When users go up one level, they find an icon that shows a cooking pan. It is animated and is interpreted by novice users as: the oven is still cooking

3. There is no tactile, visual or audible feedback that indicates that the oven stopped cooking after pressing the stop button.

\begin{tabular}{|c|c|c|}
\hline EventnoEventlog & Msec. & Video file \\
\hline $\begin{array}{l}\text { Did things...I pushed a } \\
794 \text { button...I think the oven } \\
\text { stopped }\end{array}$ & 4819 & NL281003_930_riette.avi \\
\hline 375 Goes to settings & 5156 & NL281003_1400_robert.avi \\
\hline $653 \begin{array}{l}\text { Does not see that it has } \\
\text { stopped already }\end{array}$ & 5482 & NL30102003_Marion.avi \\
\hline $\begin{array}{l}320 \text { ls it stopped...ggoes to } \\
\text { start now }\end{array}$ & 6025 & NL031103_1400_Femke.avi \\
\hline $\begin{array}{l}\text { What do you think when } \\
518 \text { you saw this... what } \\
\text { should I do now }\end{array}$ & 6244 & NL04112003_900_karin_1.avi \\
\hline 481Tries to turn & 6749 & NL051103_1400_wim.avi \\
\hline
\end{tabular}

\section{Weight:}

Weight $=$ high. In emergency situations novice users will not be sure whether the oven stopped and especially in dangerous situations they may try to stop it again or to verify whether it stopped.

\section{Suggestions:}

1. Give feedback when stopping the oven, for example a small screen indicating that the oven stopped. Or an indicator of the on/off status of the oven that can be seen in each menu. Suppose that the oven evolves and users can change settings without stopping cooking, such an indicator maybe very useful (see findings on alarm-clock)

2. The word "cooking" is very active: it seems to indicate the status of the oven. Maybe there is an alternative word. After selecting suggestion 1 the confusion is probably already over.

3. Some users preferred a stop/start button. This is also a way of providing immediate feedback: a clear start/stop button that is in or out depending on the status of the oven. 
Appendix 4 Examples from lab C's report (lab specific analysis).

\section{Problems and issues with control panel listed by task}

This section describes the main problems identified during observation of the sample users. Suggestions for change are given in the tables below. More detailed recommendations for change listed per screen are shown in Appendix 1.

Scenario 1: Stop the cooking

\begin{tabular}{|l|l|}
\hline Problem/comment & Recommendation \\
\hline $\begin{array}{l}\text { There is no clear feedback after pressing 'stop', } \\
\text { the cooking process has stopped. 7 users } \\
\text { continued interacting thinking that oven was still } \\
\text { cooking. One user tried to reduce the temperature } \\
\text { while another thought oven temperature would } \\
\text { show it cooling. }\end{array}$ & $\begin{array}{l}\text { After pressing stop, user should go to a screen that } \\
\text { indicates stop and gives the options to 'stop } \\
\text { completely' or 'continue' (default - stop } \\
\text { completely). After stop, system should return to } \\
\text { main menu. }\end{array}$ \\
\hline $\begin{array}{l}\text { When user gets back to main menu, the current } \\
\text { option is 'cooking', which indicates that the oven } \\
\text { is still cooking. }\end{array}$ & Maybe re-label the tab as 'cook' or 'cook setup' \\
\hline $\begin{array}{l}\text { Left hand arrow indicating that there is a submenu } \\
\text { available is not clear. }\end{array}$ & $\begin{array}{l}\text { Consider another way to indicate lower level } \\
\text { menu, e.g. place arrow after the menu option, or } \\
\text { place 3 dots after the menu option. }\end{array}$ \\
\hline
\end{tabular}

(From the report's appendix 1:)

$<$ screen image that is shown after the cooking process has stopped>
- After the user presses Stop, there is no feedback that the oven has stopped cooking. Pressing Stop should lead to a screen with a heading and two options:

\section{COOKING PAUSED}

\section{Stop completely}

Continue

This would give the user the option to either stop completely, or to check the food and continue. If the user presses 'Stop completely' then the cooker should return to the Main Menu. 
Appendix 5 Example of a log made by lab A (SlimDEVAN analysis).

\begin{tabular}{|c|c|c|}
\hline \multicolumn{3}{|c|}{ USER 7: FEMALE, 41, HIGH INCOME } \\
\hline $\begin{array}{l}\text { Actions } \\
\text { (time stamp) } \\
(00: 27)\end{array}$ & $\begin{array}{l}\text { Verbal utterances, user } \\
\text { behaviour }\end{array}$ & $\begin{array}{l}\text { Breakdown signal types } \\
\text { TASK: Stop the oven cooking }\end{array}$ \\
\hline $\begin{array}{l}\text { presses rotary knob } \\
(00: 28)\end{array}$ & Shall I stop now? & ACT \\
\hline $\begin{array}{l}\text { turns rotary knob } \\
(00: 29)\end{array}$ & & ACT \\
\hline $\begin{array}{l}\text { Repeated turning and pressing } \\
\text { rotary knob } \\
<0: 29: 00: 32>\end{array}$ & & REP, ACT \\
\hline$(00: 33)$ & Didn’t stop! & $\begin{array}{l}\text { RAND and DSF } \\
\text { RAND: After the end of the task it } \\
\text { was indicated by the user that she } \\
\text { hadn't seen stop button, she had } \\
\text { pressed it unintentionally. } \\
\text { DSF: Surprized by the action's } \\
\text { effect, can't understand why she } \\
\text { couldn't stop }\end{array}$ \\
\hline $\begin{array}{l}\text { TASK GOAL ACHIEVED } \\
(00: 34)\end{array}$ & & $\begin{array}{l}\text { DSF } \\
\text { Doesn't understand action's effect }\end{array}$ \\
\hline $\begin{array}{l}(00: 35) \\
\text { cooking diplay appears }\end{array}$ & Himm... & $\begin{array}{l}\text { DSF } \\
\text { Surprised by the action's effect }\end{array}$ \\
\hline$(00: 43)$ & Now select something ?..... & $\begin{array}{l}\text { INTN } \\
\text { Specifies an action that she thinks is } \\
\text { needed }\end{array}$ \\
\hline$(00: 44)$ & What shall I do? & $\begin{array}{l}\text { PUZZ } \\
\text { Doesn't know what action is needed } \\
\text { to stop }\end{array}$ \\
\hline$(00: 53)$ & Didn't stop! & $\begin{array}{l}\text { WEX } \\
\text { Oven has already stopped but she } \\
\text { formulates a wrong explanation for } \\
\text { the display }\end{array}$ \\
\hline
\end{tabular}


Appendix 6. Example of usability problem list as reported by lab A (SlimDEVAN analysis).

\begin{tabular}{|c|c|c|}
\hline \multicolumn{3}{|c|}{$<$ Name $>$} \\
\hline $\begin{array}{ll}\text { Time } & \text { stamp } \\
\text { and } & \text { signal } \\
\text { codes } & \\
\end{array}$ & $\begin{array}{l}\text { Free-form } \\
\text { description }\end{array}$ & $\begin{array}{l}\text { Inferences about what design } \\
\text { elements may have caused the } \\
\text { difficulties to occur. }\end{array}$ \\
\hline $\begin{array}{l}(00: 28) \\
\text { ACT }\end{array}$ & $\begin{array}{l}\text { User presses rotary knob to } \\
\text { stop the oven }\end{array}$ & $\begin{array}{l}\text { The rotary knob is the most dominant } \\
\text { element among the controls, so that user } \\
\text { is directed to that without much } \\
\text { intention. Furthermore, stop is not } \\
\text { sufficiently expressed. }\end{array}$ \\
\hline $\begin{array}{l}<0: 29: \\
00: 32> \\
\text { REP, ACT }\end{array}$ & $\begin{array}{l}\text { User rotates and presses the } \\
\text { knob several times to stop. }\end{array}$ & $\begin{array}{l}\text { User expects to control the oven with } \\
\text { the knob, just as she uses a conventional } \\
\text { oven. This is a problem regarding user's } \\
\text { expectation. }\end{array}$ \\
\hline $\begin{array}{l}(00: 53) \\
\text { WEX }\end{array}$ & $\begin{array}{l}\text { Although she successfully } \\
\text { stopped the oven she } \\
\text { expresses that she was not } \\
\text { successful. }\end{array}$ & $\begin{array}{l}\text { After the oven is stopped user is not } \\
\text { informed. The info present in the } \\
\text { following screen actually misleads the } \\
\text { user. }\end{array}$ \\
\hline
\end{tabular}


Appendix 7. Example of part of a session log made by lab B (SlimDEVAN analysis). Columns (from left to right): time and date; logged events; code indicating participant; task; video frame number; reference to video file.

\begin{tabular}{|r|l|r|l|r|l|}
\hline & $\begin{array}{l}\text { [SYS] [switched to task Stop the } \\
\text { cooking] }\end{array}$ & 37 & Stop the cooking & 289 & \\
\hline $\begin{array}{r}28-10-2003 \\
14: 15\end{array}$ & $\ldots$. go ahead & 37 & Stop the cooking & 344 & NL281003_1400_robert.avi \\
\hline $\begin{array}{r}28-10-2003 \\
14: 15\end{array}$ & this is the oven & 37 & Stop the cooking & 349 & NL281003_1400_robert.avi \\
\hline $\begin{array}{r}28-10-2003 \\
14: 15\end{array}$ & presses...loooks, turns the button & 37 & Stop the cooking & 352 & NL281003_1400_robert.avi \\
\hline & $\begin{array}{l}\text { [DEVAN] [ACT], Wrong Action: } \\
\text { presses...loooks, turns the button }\end{array}$ & 37 & Stop the cooking & 357 & NL281003_1400_robert.avi \\
\hline $\begin{array}{r}28-10-2003 \\
14: 15\end{array}$ & presses at back & 37 & Stop the cooking & 360 & NL281003_1400_robert.avi \\
\hline $\begin{array}{r}28-10-2003 \\
14: 15\end{array}$ & goes to menu & 37 & Stop the cooking & 363 & NL281003_1400_robert.avi \\
\hline $\begin{array}{r}28-10-2003 \\
14: 15\end{array}$ & recipes & 37 & Stop the cooking & 369 & NL281003_1400_robert.avi \\
\hline $\begin{array}{r}\text { [DEVAN] [ACT], Wrong Action: } \\
\text { recipes }\end{array}$ & 37 & Stop the cooking & 369 & NL281003_1400_robert.avi \\
\hline $14: 15$ & goes to settings & 37 & Stop the cooking & 375 & NL281003_1400_robert.avi \\
\hline $\begin{array}{r}28-10-2003 \\
14: 15\end{array}$ & alarm & 37 & Stop the cooking & 378 & NL281003_1400_robert.avi \\
\hline $\begin{array}{r}28-10-2003 \\
14: 15\end{array}$ & status of alarm & 37 & Stop the cooking & 380 & NL281003_1400_robert.avi \\
\hline $\begin{array}{r}28-10-2003 \\
14: 15\end{array}$ & cooking & 37 & Stop the cooking & 383 & NL281003_1400_robert.avi \\
\hline $\begin{array}{r}28-10-2003 \\
14: 15\end{array}$ & turns knob & 37 & Stop the cooking & 389 & NL281003_1400_robert.avi \\
\hline $\begin{array}{r}28-10-2003 \\
14: 15\end{array}$ & I'm looking for a stopbutton & 37 & Stop the cooking & 394 & NL281003_1400_robert.avi \\
\hline & $\begin{array}{l}\text { [DEVAN] [SEARCH], Searches for } \\
\text { Function: Im looking for a } \\
\text { stopbutton }\end{array}$ & 37 & Stop the cooking & 394 & NL281003_1400_robert.avi \\
\hline $\begin{array}{r}28-10-2003 \\
14: 16\end{array}$ & I can't find it & 37 & Stop the cooking & 402 & NL281003_1400_robert.avi \\
\hline & & & & \\
\hline
\end{tabular}


Appendix 8. Parts from the usability problem report of lab $B$ (SlimDEVAN analysis).

\section{Usability issues related to the cooking sereens.}

MAIN

1. When alarm is set it is not clear if time indicates time until alarm or indicates the actual time

STARTING/STOPPING

1. All users press the stop button when asked to stop the cooking process. However: the feedback is not clear. The "cooking" title above the menu, the animated and moving "Cooking"-picture suggest that the oven is still operating.

2. Cooking is interpreted as "Boiling". The animated gif with the boiling pan supports this interpretation.

It is better to have a picture of an oven.

Weight

The issues related to starting and stopping the oven are found several times in each session (in total 34 The is times)

The issues related to editing were found 16 times, and in each session. The issues related to the menu, status were found in at least three sessions. The defrost issues were found in two sessions.

The start/stop issues are therefore probably quite general for the user population, also the issues related to the edit-menu. The issues related to status, menu and defrost were found in specific sessions in which users were less experienced.

\section{Validity}

Start and stop issues and issues related to the edit cooking are quite central to the operation of the oven. In general it can be concluded that users can learn to operate the oven, without a manual but they need some learning time. Reaching errorless and routine performance will probably take some time, for the less used functions it may be difficult to reach.

\section{Suggestions}

1. If applicable, present In the opening screen also information on selected program and alarm settings. 2. The term "Cooking" is confusing. Although it finally remains the only option for setting settings after users have tried the other items (setting duration in the time dialog or in the alarm dialog, looking in recipes). The icon is associated with boiling (potatoes, vegetables) and not with an oven.

The cooking icon is animated, suggesting that the oven is actually working. Also the title "cooking" suggests The cooking icon is animated, suggesting that the oven is actually working. Also the title "cooking" suggests
that the oven is working. A clear indication of the status of the oven, a program or alarm could help users to understand what the oven is doing when they are in the main menu.

3. Pressing the stop-button should give feedback, for instance a sound.

FINDING 641 D_cooking_adaptation of parameters: When changing duration the old temperature is not shown. If user forgets: cannot cancel changes. Not clear if duration indicates minutes to go or total duration

[DEVAN][COMM], Comment ... He is on bottom now... it works...the minutes I changed...I suppose it is clear.... is not sure if minutes where actually changed (program does not take change in time !!!)

Pre-Interview

video

1259

[DEVAN][COMM], Comment. was not clear that minutes were added... I was not sure if duration was the total time or the time left

$\begin{array}{llll}\text { session: } & 41 & \text { Prolong the cooking time video } & 1280\end{array}$

[DEVAN][DIFF], Execution Difficulty: .... Im not sure I forgot to check how mary minuytes to go...

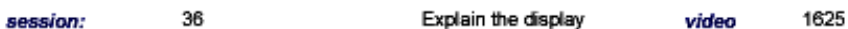


Appendix 9. Example from a log file made by lab C (SlimDEVAN analysis).

TEBA OVEN STUDY

SUBJECT 5: <NAME>

\begin{tabular}{|c|c|c|}
\hline $\begin{array}{l}\text { Actions/Times } \\
\text { (minutes and seconds) }\end{array}$ & $\begin{array}{l}\text { Verbal utterances/User } \\
\text { behaviour }\end{array}$ & Difficulty signals \\
\hline 0.11 Pressed STOP. & $\begin{array}{l}\text { "The natural thing would } \\
\text { be to press the button that } \\
\text { says Stop. But I don't think } \\
\text { that's right. Perhaps I'll } \\
\text { twiddle that knob." }\end{array}$ & $\begin{array}{l}\text { Task 1: Stop the cooker } \\
\text { User achieves task but } \\
\text { does not realise it. }\end{array}$ \\
\hline $\begin{array}{l}0.17 \text { Tries out knob and } \\
\text { moves up to MODE. }\end{array}$ & $\begin{array}{l}\text { "But that doesn't do } \\
\text { anything. }\end{array}$ & \\
\hline $\begin{array}{l}0.30 \text { Down to DURATION } \\
\text { and up to MODE. }\end{array}$ & "So..." & $\mathrm{ACT}$ \\
\hline 0.32 Selects MODE & & $\mathrm{ACT}$ \\
\hline $\begin{array}{l}0.36 \text { Twiddles knob within } \\
\text { mode options. }\end{array}$ & & $\mathrm{ACT}$ \\
\hline $\begin{array}{l}\text { 0.39 Presses BACK to go to } \\
\text { MODE }\end{array}$ & $\begin{array}{l}\text { "The oven's going to blow } \\
\text { up I suppose. I'm stuck. I } \\
\text { don't know what to do. I've } \\
\text { probably messed it up." }\end{array}$ & PUZZ \\
\hline 0.44 Task completed & & \\
\hline
\end{tabular}


Appendix 10. Example from the problem list made by lab $C$. (SlimDEVAN analysis).

\author{
Problem list following by subjects who experienced them (numbered 1 to 8 ). \\ Lack of feedback when stopping oven $(1,2,3,4,5,6,7,8)$ \\ Fixed order of making settings in cooking menu $(1,2,3,4,5,6,7)$ \\ Hesitating or not realising that they should select COOK to start cooking $(1,2,3,4,5,6,7,8)$ \\ Expects selection bar in View and Cook screen (7) \\ Did not realize had to press VIEW AND COOK (4)
}

